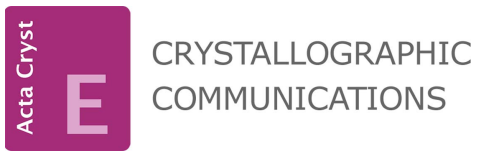

ISSN 2056-9890

Received 23 January 2015

Accepted 7 February 2015

Edited by S. Parkin, University of Kentucky, USA

Keywords: crystal structure; platinum-

containing heteropolyoxomolybdate; centrosymmetric hydrogen bond; strong hydrogen bond; Anderson-type heteropolyoxomolybdate

CCDC reference: 1048266

Supporting information: this article has

supporting information at journals.iucr.org/e

\section{Crystal structure of heptaguanidinium nonahydrogen bis[a-hexamolybdoplatinate(IV)] heptahydrate}

\author{
Hea-Chung Joo, ${ }^{a}$ Ki-Min Park ${ }^{\mathrm{b}}$ and Uk Lee ${ }^{\mathrm{a} *}$
}

\begin{abstract}
${ }^{a}$ Department of Chemistry, Pukyong National University, 599-1 Daeyeon 3-dong, Nam-gu, Busan 608-737, Republic of Korea, and ${ }^{\mathbf{b}}$ The Research Institute of Natural Science, Gyeongsan National University, Jinju 660-701, Republic of Korea. *Correspondence e-mail: uklee@pknu.ac.kr
\end{abstract}

The title compound, $\left(\mathrm{CH}_{6} \mathrm{~N}_{3}\right)_{7} \mathrm{H}_{9}\left[\mathrm{PtMo}_{6} \mathrm{O}_{24}\right]_{2} \cdot 7 \mathrm{H}_{2} \mathrm{O}$, containing the well-known Anderson-type heteropolyoxomolybdate, was obtained by recrystallization of its powdered guanidinium salt. The protonated $\mathrm{O}$ atoms in the polyanion were confirmed by electron-density maps, interpolyanion hydrogen bonds and bondvalance sums (BVS). The $\left\{\left[\mathrm{H}_{4.5} \mathrm{PtMo}_{6} \mathrm{O}_{24}\right]_{2}\right\}^{7-}$ polyanion is the same as that already characterized in $\mathrm{K}_{7}\left[\mathrm{H}_{4.5} \mathrm{PtMo}_{6} \mathrm{O}_{24}\right]_{2} \cdot 11 \mathrm{H}_{2} \mathrm{O}$ [space group $P \overline{1}$; Lee \& Joo (2010). Acta Cryst. E66, i8-i9]. The heteropolyanions form inversion-generated dimers, $\left\{\left[\mathrm{H}_{4.5} \mathrm{PtMo}_{6} \mathrm{O}_{24}\right]_{2}\right\}^{7-}$, held together by each of the four $\mu_{3}-\mathrm{O}-\mathrm{H} \cdots \mu_{1}-\mathrm{O}$, two $\mu_{2}-\mathrm{O}-\mathrm{H} \cdots \mu_{2}-\mathrm{O}$ hydrogen bonds and one centrosymmetric $\mu_{3}-\mathrm{O}-\mathrm{H}-\mu_{3}-\mathrm{O}$ hydrogen bond. The $\mathrm{H}$ atom of the centrosymmetric hydrogen bond is located on an inversion centre. One guanidinium ion and one water molecule are equally disordered about a twofold rotation axis.

\section{Chemical context}

The $\alpha$ (planar structure)- $\beta$ (bent structure)- $\alpha$ geometrical isomerization, according to stepwise protonation in the $\left[\mathrm{PtMo}_{6} \mathrm{O}_{24}\right]^{8-}$ polyoxometalate (POM) species, viz. $\left(\left[\mathrm{H}_{3.5} \alpha-\right.\right.$ $\left.\mathrm{PtMo}_{6} \mathrm{O}_{24}\right]^{4.5-}$ (Lee \& Sasaki, 1994), $\left[\mathrm{H}_{4} \beta-\mathrm{PtMo}_{6} \mathrm{O}_{24}\right]^{4-}$ (Lee \& Sasaki, 1994; Joo et al., 1994) and $\left[\mathrm{H}_{4.5} \alpha-\mathrm{PtMo}_{6} \mathrm{O}_{24}\right]^{3.5-}$ (Lee \& Sasaki, 1994; Lee et al., 2010) is an unprecedented phenomenon in the Anderson-type heteropolyanion (Anderson, 1937), as well as in the chemistry of polyoxometalates.

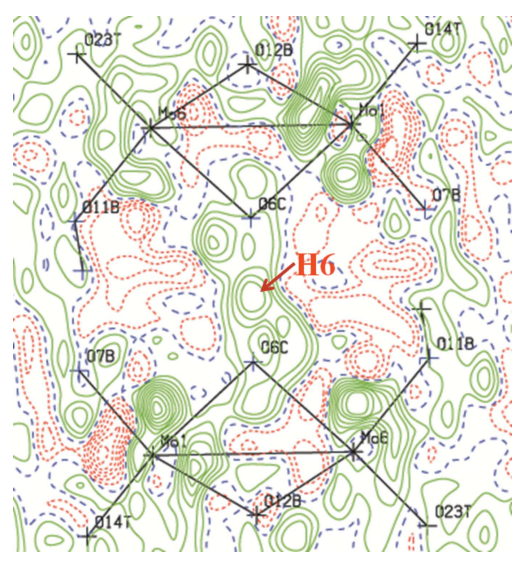

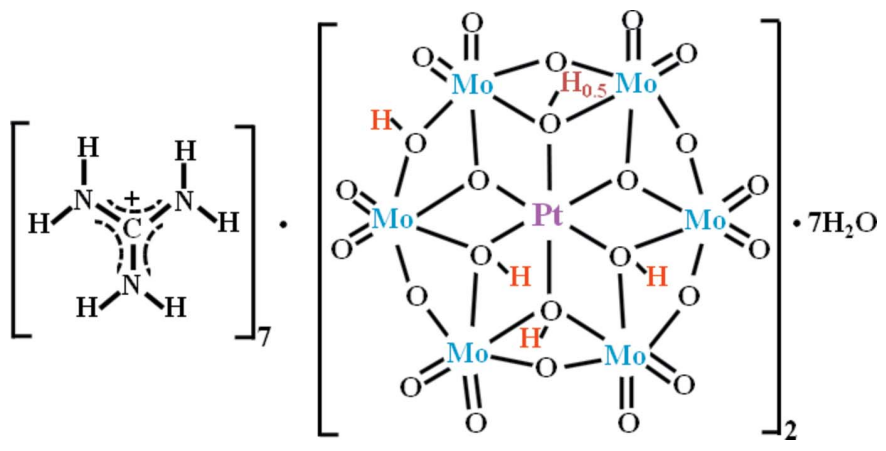

As a result of the insolubility of the guanidinium salt, replaceable counter-cations in POMs can be exchanged by guanidinium ions. It is thus possible to obtain stable POMs by precipitation from aqueous solution with guanidinium salts. The guanidinium salts of platinum-containing POM species, viz. $\left(\mathrm{CH}_{6} \mathrm{~N}_{3}\right)_{8}\left[\mathrm{PtW}_{6} \mathrm{O}_{24}\right]$ (Lee et al., 2003), $\left(\mathrm{CH}_{6} \mathrm{~N}_{3}\right)_{5^{-}}$ 


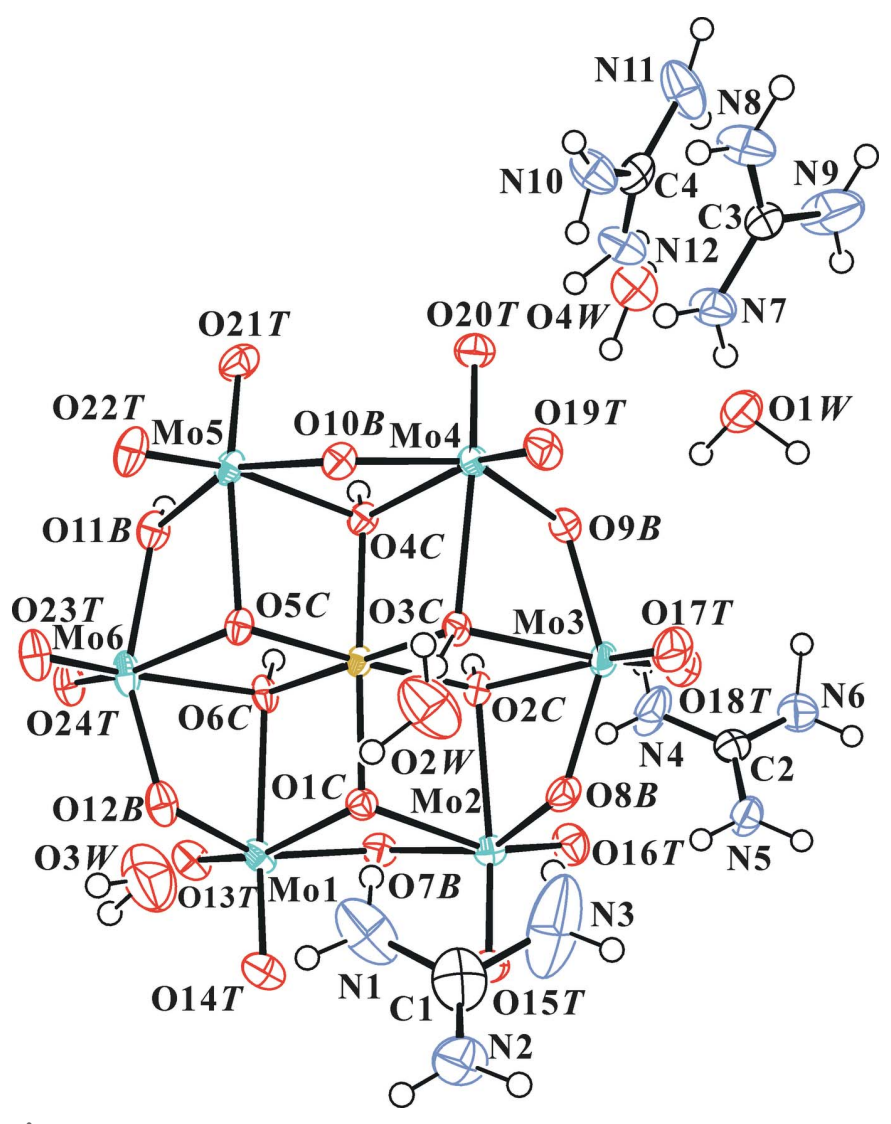

Figure 1

The molecular structure of the title compound, showing the atomnumbering scheme. Displacement ellipsoids are drawn at the $50 \%$ probability level. $\mathrm{H}$ atoms are presented as small spheres of arbitrary radius. Disordered parts have been omitted for clarity.

$\left[\mathrm{H}_{2} \mathrm{PtV}_{9} \mathrm{O}_{28}\right]$ (Joo et al., 2011) and $\left(\mathrm{CH}_{6} \mathrm{~N}_{3}\right)_{8}\left[\alpha-\mathrm{SiPt}_{2^{-}}\right.$ $\left.\mathrm{W}_{10} \mathrm{O}_{40}\right] \cdot 6 \mathrm{H}_{2} \mathrm{O}$ (Lee et al., 2003) have been reported by our group. The positions of the protonated $\mathrm{O}$ atoms in the $\left\{\left[\mathrm{H}_{4.5} \alpha-\mathrm{PtMo}_{6} \mathrm{O}_{24}\right]_{2}\right\}^{7-}$ polyanion were reconfirmed in the present study.

Sometimes a short hydrogen bond, O $\cdots \mathrm{O}$ distance $<2.60 \AA$, in which the $\mathrm{H}$ atom lies on a crystallographic center of symmetry, occurs in this class of structure. The $\mathrm{H}$ atom of the central hydrogen bond, $\mathrm{O} 6 \mathrm{C}-\mathrm{H} 6-\mathrm{O} 6 C^{\mathrm{i}}$ in the title compound lies on a crystallographic center of symmetry (space group $C 2 / c: \frac{3}{4}, \frac{1}{4}, \frac{1}{2}$ ).

\section{Structural commentary}

The structure of the title compound POM anion has been discussed in detail (Lee et al., 2010). Fig. 1 shows the structure of the title compound, and selected geometrical parameters are given in Table 1 . The complete polyanion has $C_{1}$ (1) symmetry. The $\mathrm{O}$ atoms of the heteropolyanion have been designated as $\mathrm{O} T$ (terminal $\mathrm{Mo}=\mathrm{O}$ atom), $\mathrm{OB}$ (bridging $\mu_{2}-\mathrm{O}$ atom), and $\mathrm{OC}$ (centered $\mu_{3}-\mathrm{O}$ atom). The protonated $\mathrm{O}$ atoms in the polyanion were confirmed in electron density maps, interpolyanion hydrogen bonds (Table 2) and by bondvalence sums (BVS; Brown \& Altermatt, 1985; Brese \&
Table 1

Selected geometric parameters $\left(\AA,{ }^{\circ}\right)$.

\begin{tabular}{|c|c|c|c|}
\hline $\mathrm{Pt} 1-\mathrm{O} 1 C$ & $1.995(3)$ & $\mathrm{Mo5}-\mathrm{O} 5 \mathrm{C}$ & $2.178(3)$ \\
\hline $\mathrm{Pt} 1-\mathrm{O} 2 \mathrm{C}$ & $2.015(3)$ & Mo6-O5C & $2.123(3)$ \\
\hline $\mathrm{Pt} 1-\mathrm{O} 3 \mathrm{C}$ & $2.027(3)$ & Mo6-O6C & $2.277(3)$ \\
\hline $\mathrm{Pt} 1-\mathrm{O} 4 \mathrm{C}$ & $2.011(3)$ & Mo1-O7B & $1.965(3)$ \\
\hline $\mathrm{Pt} 1-\mathrm{O} 5 \mathrm{C}$ & $1.997(3)$ & Mo1-O12B & $1.959(3)$ \\
\hline $\mathrm{Pt} 1-\mathrm{O} 6 \mathrm{C}$ & $2.005(3)$ & Mo2-O7B & $1.978(3)$ \\
\hline $\mathrm{Mo} 1-\mathrm{O} 1 C$ & $2.150(3)$ & Mo2-O8B & $1.945(3)$ \\
\hline Mo1-O6C & $2.317(3)$ & $\mathrm{Mo3}-\mathrm{O} 8 B$ & $1.934(3)$ \\
\hline $\mathrm{Mo} 2-\mathrm{O} 1 C$ & $2.248(3)$ & Mo3-O9B & $1.952(3)$ \\
\hline $\mathrm{Mo} 2-\mathrm{O} 2 C$ & $2.286(3)$ & Mo4-O9B & $1.941(3)$ \\
\hline $\mathrm{Mo} 3-\mathrm{O} 2 \mathrm{C}$ & $2.307(3)$ & Mo4-O10B & $1.959(3)$ \\
\hline $\mathrm{Mo} 3-\mathrm{O} 3 C$ & $2.318(3)$ & Mo5-O10B & $1.895(3)$ \\
\hline $\mathrm{Mo} 4-\mathrm{O} 3 C$ & $2.287(3)$ & Mo5-O11B & $2.058(3)$ \\
\hline $\mathrm{Mo} 4-\mathrm{O} 4 C$ & $2.327(3)$ & Mo6-O11B & $2.075(4)$ \\
\hline $\mathrm{Mo} 5-\mathrm{O} 4 C$ & $2.289(3)$ & Mo6-O12B & $1.894(4)$ \\
\hline Mo1-O1C-Mo2 & $95.79(12)$ & $\mathrm{Mo} 1-\mathrm{O} 7 \mathrm{~B}-\mathrm{Mo} 2$ & $111.71(15)$ \\
\hline $\mathrm{Mo} 2-\mathrm{O} 2 C-\mathrm{Mo} 3$ & $93.64(11)$ & Mo3-O8B-Mo2 & $119.36(16)$ \\
\hline $\mathrm{Mo} 4-\mathrm{O} 3 C-\mathrm{Mo} 3$ & $93.75(12)$ & Mo4-O9B-Mo3 & $119.39(17)$ \\
\hline $\mathrm{Mo5}-\mathrm{O} 4 C-\mathrm{Mo} 4$ & 92.64 (11) & Mo5-O10B-Mo4 & $120.02(16)$ \\
\hline Mo6-O5C-Mo5 & $102.87(13)$ & Mo5-O11B-Mo6 & $108.97(15)$ \\
\hline Mo6-O6C-Mo1 & $91.14(12)$ & Mo6-O12B-Mo1 & $116.75(17)$ \\
\hline
\end{tabular}

Table 2

Hydrogen-bond geometry $\left(\AA{ }^{\circ}\right)$.

\begin{tabular}{|c|c|c|c|c|}
\hline$D-\mathrm{H} \cdots A$ & $D-\mathrm{H}$ & $\mathrm{H} \cdots A$ & $D \cdots A$ & $D-\mathrm{H} \cdots A$ \\
\hline $\mathrm{O} 2 C-\mathrm{H} 2 \cdots \mathrm{O} 24 T^{\mathrm{i}}$ & $0.96(2)$ & $1.61(2)$ & $2.578(5)$ & $179(6)$ \\
\hline $\mathrm{O} 3 C-\mathrm{H} 3 \cdots \mathrm{O} 2 W$ & $0.96(2)$ & $1.69(3)$ & $2.622(6)$ & $164(7)$ \\
\hline $\mathrm{O} 4 C-\mathrm{H} 4 \cdots \mathrm{O} 13 T^{\mathrm{i}}$ & $0.95(2)$ & $1.63(2)$ & $2.568(5)$ & $173(9)$ \\
\hline $\mathrm{O} 6 C-\mathrm{H} 6 \cdots \mathrm{O} 6 C^{\mathrm{i}}$ & 1.27 & 1.27 & $2.532(6)$ & 180 \\
\hline $\mathrm{O} 11 B-\mathrm{H} 11 \cdots \mathrm{O} 7 B^{\mathrm{i}}$ & $0.95(2)$ & $1.74(2)$ & $2.679(5)$ & $173(10)$ \\
\hline $\mathrm{N} 1-\mathrm{H} 1 B \cdots \mathrm{O} 1 C$ & 0.88 & 2.05 & $2.864(6)$ & 154 \\
\hline $\mathrm{N} 1-\mathrm{H} 1 A \cdots \mathrm{O} 3 W$ & 0.88 & 2.33 & $2.973(9)$ & 130 \\
\hline $\mathrm{N} 2-\mathrm{H} 2 A \cdots \mathrm{O} 18 T^{\mathrm{ii}}$ & 0.88 & 2.08 & $2.940(7)$ & 165 \\
\hline $\mathrm{N} 2-\mathrm{H} 2 B \cdots \mathrm{O} 19 T^{\mathrm{iii}}$ & 0.88 & 2.22 & $3.043(6)$ & 155 \\
\hline $\mathrm{N} 3-\mathrm{H} 3 B \cdots \mathrm{O} 8 B$ & 0.88 & 2.04 & $2.874(7)$ & 157 \\
\hline $\mathrm{N} 3-\mathrm{H} 3 A \cdots \mathrm{O} 2 W^{\mathrm{iii}}$ & 0.88 & 2.25 & $2.979(9)$ & 140 \\
\hline $\mathrm{N} 4-\mathrm{H} 4 B \cdots \mathrm{O} 14 T^{\mathrm{iv}}$ & 0.88 & 2.09 & $2.944(6)$ & 164 \\
\hline $\mathrm{N} 4-\mathrm{H} 4 A \cdots \mathrm{O} 24 T^{\mathrm{i}}$ & 0.88 & 2.48 & $3.006(6)$ & 119 \\
\hline $\mathrm{N} 5-\mathrm{H} 5 A \cdots \mathrm{O} 16 T$ & 0.88 & 2.06 & $2.890(6)$ & 157 \\
\hline $\mathrm{N} 5-\mathrm{H} 5 B \cdots \mathrm{O} 21 T^{\mathrm{v}}$ & 0.88 & 2.18 & $2.973(5)$ & 149 \\
\hline $\mathrm{N} 6-\mathrm{H} 6 A \cdots \mathrm{O} 15 T^{\mathrm{iv}}$ & 0.88 & 2.19 & $2.894(6)$ & 136 \\
\hline $\mathrm{N} 6-\mathrm{H} 6 B \cdots \mathrm{O} 21 T^{\mathrm{v}}$ & 0.88 & 2.59 & $3.281(6)$ & 136 \\
\hline $\mathrm{N} 7-\mathrm{H} 7 B \cdots \mathrm{O} 19 T$ & 0.88 & 2.40 & $2.936(5)$ & 119 \\
\hline $\mathrm{N} 7-\mathrm{H} 7 A \cdots \mathrm{O} 1 W$ & 0.88 & 2.11 & $2.927(6)$ & 154 \\
\hline $\mathrm{N} 8-\mathrm{H} 8 B \cdots \mathrm{O} 13 T^{\mathrm{vi}}$ & 0.88 & 2.39 & $3.006(6)$ & 128 \\
\hline $\mathrm{N} 8-\mathrm{H} 8 A \cdots \mathrm{O} 23 T^{\mathrm{vii}}$ & 0.88 & 2.04 & $2.918(6)$ & 178 \\
\hline $\mathrm{N} 9-\mathrm{H} 9 A \cdots \mathrm{O} 22 T^{\mathrm{vii}}$ & 0.88 & 2.21 & $2.938(7)$ & 140 \\
\hline $\mathrm{O} 1 W-\mathrm{H} 1 A W \cdots \mathrm{O} 9 B$ & $0.94(2)$ & $2.20(5)$ & $2.916(5)$ & $132(5)$ \\
\hline $\mathrm{O} 1 W-\mathrm{H} 1 B W \cdots \mathrm{O} 17 T^{\mathrm{viii}}$ & $0.95(2)$ & $1.85(3)$ & $2.783(5)$ & $166(6)$ \\
\hline $\mathrm{O} 2 W-\mathrm{H} 2 B W \cdots \mathrm{O} 4 W^{\mathrm{ii}}$ & $0.95(2)$ & $2.24(7)$ & $2.902(12)$ & $126(6)$ \\
\hline $\mathrm{O} 3 W-\mathrm{H} 3 B W \cdots \mathrm{O} 9 B^{\mathrm{ii}}$ & $0.94(2)$ & $2.35(8)$ & $3.029(7)$ & $128(8)$ \\
\hline
\end{tabular}

Symmetry codes: (i) $-x+\frac{3}{2},-y+\frac{1}{2},-z+1$; (ii) $x, y-1, z$; (iii) $-x+1,-y,-z+1$; (iv) $x, y+1, z$; (v) $x,-y+1, z+\frac{1}{2}$; (vi) $x-\frac{1}{2},-y+\frac{1}{2}, z-\frac{1}{2}$; (vii) $-x+1, y+1,-z+\frac{1}{2}$; (viii) $-x+1,-y+1,-z+1$.

O'Keeffe, 1991). Fig. 2 shows a symmetric electron-density map around the position of atom H6. The $\mathrm{H}$ atom of the centrosymmetric hydrogen bond in the compound lies on a crystallographic centre of symmetry (space group $C 2 / c: \frac{3}{4}, \frac{1}{4}, \frac{1}{2}$ ). The $\mathrm{O} 6 C-\mathrm{H} 6$ and $\mathrm{O} 6 C \cdot \cdots \mathrm{O} 6 C^{\mathrm{i}}$ distances are 1.27 and 2.532 (6) $\AA$, and the $\mathrm{O} 6 C-\mathrm{H} 6-\mathrm{O}^{\circ} \mathrm{C}^{\mathrm{i}}$ angle is $180^{\circ}$ (Table 2 and Fig. 3). Atom $\mathrm{H} 3$ does not contribute to dimer formation because it is located on the other side of the polyanion. 


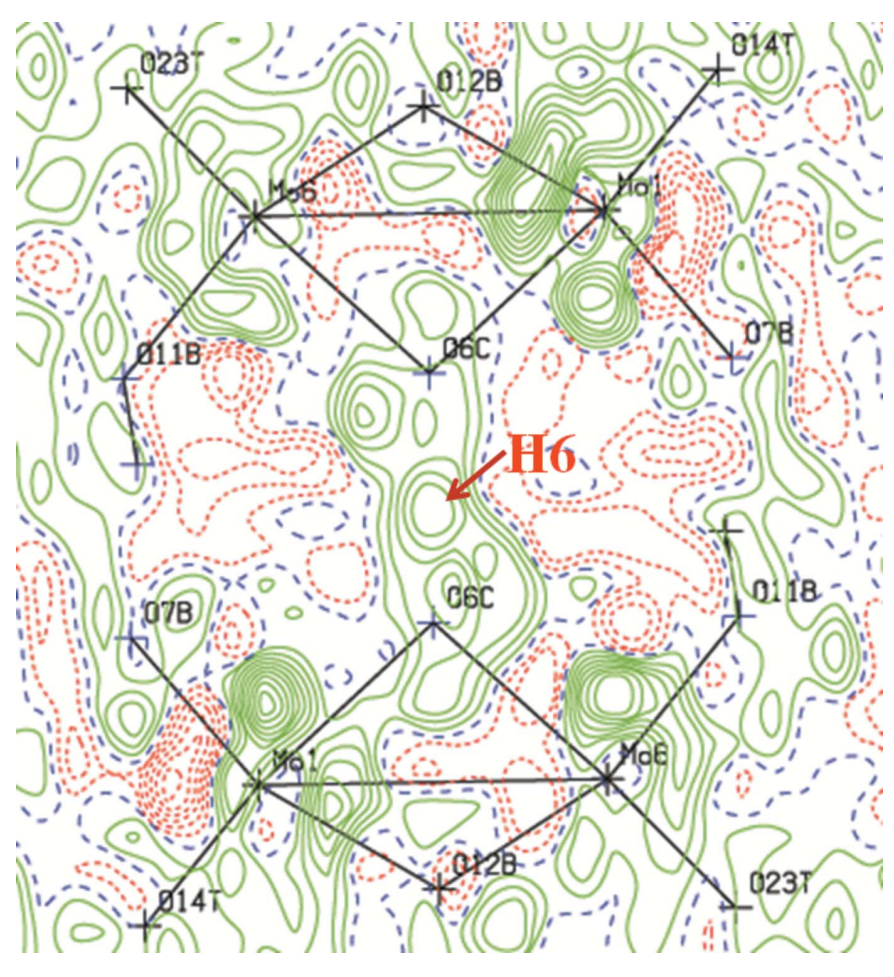

Figure 2

Difference-Fourier map around atom H6 (calculated with atom H6 absent from the model).

Confirmation of the protonated $\mathrm{O}$ atoms was strongly supported by the BVS analysis. The calculated BVS for atoms $\mathrm{O} 2 C, \mathrm{O} 3 C, \mathrm{O} 4 C, \mathrm{O} 6 C$ and $\mathrm{O} 11 B$ are $1.40,1.36,1.38,1.41$ and 1.30 valence units (v.u.), respectively, if the valence of the $\mathrm{O}-$ $\mathrm{H}$ bond is not included. Since the BVS value around the $\mu_{2}-\mathrm{O}$ atom should be 2.0 v.u., the missing valences of $\mathrm{O} 2 C, \mathrm{O} 3 C$, $\mathrm{O} 4 C, \mathrm{O} 6 C$ and $\mathrm{O} 11 B$ are $0.60,0.64,0.62,0.59$ and 0.70 v.u., respectively, which corresponds to the valence of the $\mathrm{O}-\mathrm{H}$ bonds. The BVS value range for the unprotonated $\mathrm{O} C$ and $\mathrm{O} B$ atoms is $1.68-1.90$ v.u. As a result, the protonated $\mathrm{O}$ atoms were $\mathrm{O} 2 C, \mathrm{O} 3 C, \mathrm{O} 4 C, \mathrm{O} 11 B$ and $\mathrm{O} 6 C$. The protonated features of both the $\left\{\left[\mathrm{H}_{4.5} \mathrm{PtMo}_{6} \mathrm{O}_{24}\right]_{2}\right\}^{7-}$ polyanion in the title compound and in $\mathrm{K}_{7}\left[\mathrm{H}_{4.5} \mathrm{PtMo}_{6} \mathrm{O}_{24}\right]_{2} \cdot 11 \mathrm{H}_{2} \mathrm{O}$ (space group $P \overline{1}$ ) are exactly the same. The bond lengths and bond angles involving protonated and unprotonated $\mathrm{O}$ atoms in the $\left\{\left[\mathrm{H}_{4.5} \mathrm{PtMo}_{6} \mathrm{O}_{24}\right]_{2}\right\}^{7-}$ polyanion are compared in Table 1 . The $\mathrm{Pt}-\mathrm{OC}$ bond lengths were not affected by protonation of the $\mathrm{OC}$ atoms.

The $\mathrm{C} 4$ guanidinium ion and $\mathrm{O} 4 W$ water molecule are equally disordered about a twofold rotation axis.

\section{Supramolecular features}

The heteropolyanions form inversion-generated dimers, $\left\{\left[\mathrm{H}_{4.5} \mathrm{PtMo}_{6} \mathrm{O}_{24}\right]_{2}\right\}^{7-}$ held together by each of the four $\mu_{3}-\mathrm{O}-$ $\mathrm{H} \cdots \mu_{1}-\mathrm{O}$ (terminal $\mathrm{O}$ atom), two $\mu_{2}-\mathrm{O}-\mathrm{H} \cdots \mu_{2}-\mathrm{O}$ and one centrosymmetric $\mu_{3}-\mathrm{O}-\mathrm{H}-\mu_{3}-\mathrm{O}$ hydrogen bonds (Table 2 ). Furthermore, the polyanions are linked in three dimensions via $\mathrm{N}-\mathrm{H} \cdots \mathrm{O}$ hydrogen bonds. All water molecules form

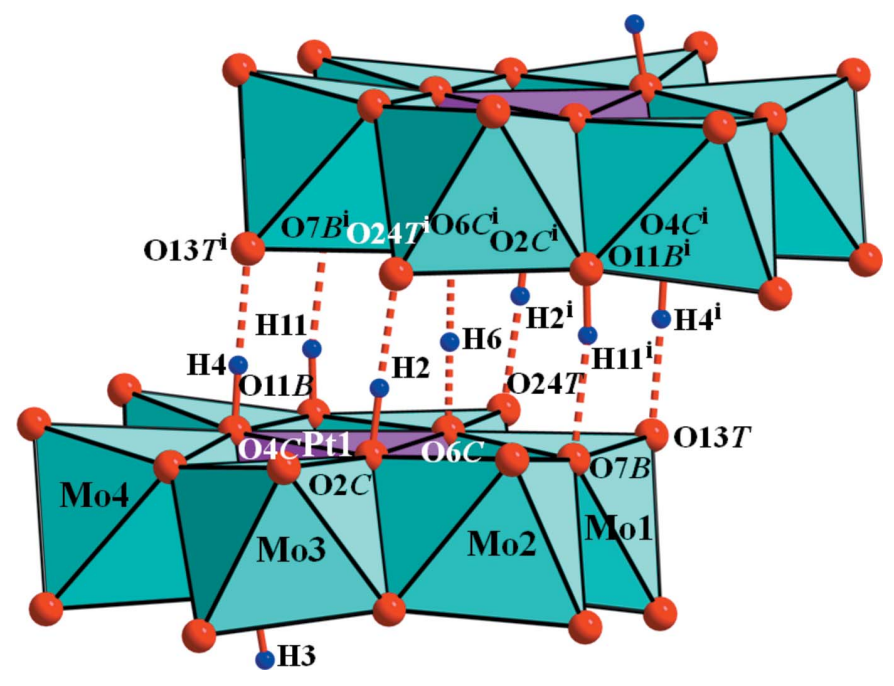

Figure 3

Polyhedral view of the heteropolyanion in the title compound with $\mathrm{O}-$ $\mathrm{H} \cdots \mathrm{O}$ contacts of the interanion hydrogen bonds shown as red dashed lines. [Symmetry code: (i) $-x+\frac{3}{2},-y+\frac{1}{2},-z+1$.]

hydrogen bonds with $\mathrm{O}$ atoms of the polyanions except for the $\mathrm{O} 2 W$ water molecule (Table 2). Hydrogen-bonding interactions involving the disordered molecules have been omitted.

\section{Database survey}

A number of Anderson-structure platinum(IV)-containing heteropolyoxomolybdates have been reported: $\left[\mathrm{H}_{4.5} \mathrm{Pt}\right.$ $\left.\mathrm{Mo}_{6} \mathrm{O}_{24}\right]^{3.5-}$ and $\left[\mathrm{H}_{4} \mathrm{PtMo}_{6} \mathrm{O}_{24}\right]^{4-},\left[\mathrm{H}_{3.5} \mathrm{PtMo}_{6} \mathrm{O}_{24}\right]^{4.5-}(\mathrm{Lee} \mathrm{\&}$ Sasaki, 1994); $\left[\mathrm{H}_{4} \beta-\mathrm{PtM0}_{6} \mathrm{O}_{24}\right]^{4-}$ (Joo et al., 1994); $\left[\mathrm{H}_{2} \mathrm{PtMo}_{6} \mathrm{O}_{24}\right]^{6-}$ (Lee \& Joo, 2000, 2004); $\left[\mathrm{H}_{4.5} \mathrm{PtMo}_{6} \mathrm{O}_{24}\right]^{3.5-}$ (Lee et al., 2010); $\left[\mathrm{H}_{6} \mathrm{PtMo}_{6} \mathrm{O}_{24}\right]^{2-}$ (Lee \& Joo, 2010); $\left[\mathrm{H}_{23}\left(\mathrm{PtMo}_{6} \mathrm{O}_{24}\right)_{4}\right]^{9-}, \quad\left[\mathrm{H}_{16}\left(\mathrm{PtMo}_{6} \mathrm{O}_{24}\right)_{3}\right]^{8-}$ and $\left[\mathrm{H}_{14^{-}}\right.$ $\left.\left(\mathrm{PtMo}_{6} \mathrm{O}_{24}\right)_{3}\right]^{14-}$ (Day et al., 2009).

\section{Synthesis and crystallization}

A pale-yellow powder of the title compound was obtained by addition of a small excess of the stoichiometric quantity of guanidinium chloride, $\mathrm{CH}_{6} \mathrm{~N}_{3} \mathrm{Cl}$, to a solution of the sodium salt of hexamolybdoplatinate hydrate. Single crystals were obtained by recrystallization from a hot aqueous solution of the crude sample in an insulating chamber.

\section{Refinement}

Crystal data, data collection and structure refinement details are summarized in Table 3. All the $\mathrm{H}$ atoms in the polyanion and all water $\mathrm{H}$ atoms were positioned using difference Fourier maps. All $\mathrm{H}$ atoms of the polyanion were refined with a distance restraint of $\mathrm{O}-\mathrm{H}=0.95$ (2) $\AA$ using the DFIX command (Sheldrick, 2008). All H atoms of the guanidinium ions were positioned geometrically and refined using a riding model, with $U_{\text {iso }}(\mathrm{H})=1.5 U_{\text {eq }}(\mathrm{N})$. The $\mathrm{C} 4$ guanidinium ion and $\mathrm{O} 4 \mathrm{~W}$ water molecule are equally disordered about a twofold rotation axis. Refinement of the site occupation factors (s.o.f) 
Table 3

Experimental details.

\begin{tabular}{|c|c|}
\hline \multicolumn{2}{|l|}{ Crystal data } \\
\hline Chemical formula & $\left(\mathrm{CH}_{6} \mathrm{~N}_{3}\right)_{7} \mathrm{H}_{9}\left[\mathrm{PtMo}_{6} \mathrm{O}_{24}\right]_{2} \cdot 7 \mathrm{H}_{2} \mathrm{O}$ \\
\hline$M_{\mathrm{r}}$ & 2865.26 \\
\hline Crystal system, space group & Monoclinic, $C 2 / c$ \\
\hline Temperature $(\mathrm{K})$ & 173 \\
\hline$a, b, c(\AA)$ & $31.413(10), 10.073(3), 23.677$ (7) \\
\hline$\beta\left({ }^{\circ}\right)$ & $119.451(14)$ \\
\hline$V\left(\AA^{6}\right)$ & $6524(3)$ \\
\hline$Z$ & 4 \\
\hline Radiation type & Mo $K \alpha$ \\
\hline$\mu\left(\mathrm{mm}^{-1}\right)$ & 6.62 \\
\hline Crystal size (mm) & $0.30 \times 0.12 \times 0.05$ \\
\hline \multicolumn{2}{|l|}{ Data collection } \\
\hline Diffractometer & Bruker SMART APEXII CCD \\
\hline Absorption correction & $\begin{array}{l}\text { Multi-scan (SADABS; Sheldrick, } \\
\text { 2008) }\end{array}$ \\
\hline$T_{\min }, T_{\max }$ & $0.241,0.729$ \\
\hline $\begin{array}{l}\text { No. of measured, independent and } \\
\text { observed }[I>2 \sigma(I)] \text { reflections }\end{array}$ & $56606,7107,6050$ \\
\hline$R_{\text {int }}$ & 0.033 \\
\hline$(\sin \theta / \lambda)_{\max }\left(\AA^{-1}\right)$ & 0.639 \\
\hline \multicolumn{2}{|l|}{ Refinement } \\
\hline$R\left[F^{2}>2 \sigma\left(F^{2}\right)\right], w R\left(F^{2}\right), S$ & $0.028,0.073,1.03$ \\
\hline No. of reflections & 7107 \\
\hline No. of parameters & 505 \\
\hline No. of restraints & 22 \\
\hline $\mathrm{H}$-atom treatment & $\begin{array}{l}\mathrm{H} \text { atoms treated by a mixture of } \\
\text { independent and constrained } \\
\text { refinement }\end{array}$ \\
\hline$\Delta \rho_{\max }, \Delta \rho_{\min }\left(\mathrm{e} \AA^{-3}\right)$ & $2.50,-1.30$ \\
\hline
\end{tabular}

Computer programs: APEX2 and SAINT (Bruker, 2009), SHELXS97 and SHELXL97 (Sheldrick, 2008), ORTEP-3 for Windows (Farrugia, 2012), PLATON (Spek, 2009) and DIAMOND (Brandenburg, 1998).

converged at values close to half occupancy. In the final refinement, the s.o.f.s were constrained to 0.5 and reasonable displacement parameters were obtained. The $\mathrm{C}-\mathrm{N}$ and $\mathrm{N}-\mathrm{H}$ bond lengths were restrained to 1.30 (2) and 0.90 (2) $\AA$, respectively, and the $\mathrm{H} A-\mathrm{N}-\mathrm{H} B$ angles were restrained by restraining the $\mathrm{H} A \cdots \mathrm{H} B$ distance to 1.55 (2) $\AA$ in the disordered $\mathrm{C} 4$ guanidinium ion using the DFIX command. The $\mathrm{H}$ atoms of all water molecules $(\mathrm{OW})$ were refined with a distance restraint of $\mathrm{O}-\mathrm{H}=0.95$ (2) $\AA$ using the DFIX, and were included in the refinement with $U_{\text {iso }}(\mathrm{H})=1.5 U_{\text {eq }}(\mathrm{O})$. The highest peak in the difference map is $0.98 \AA$ from atom Pt1 and the largest hole is $0.36 \AA$ from N3.

\section{Acknowledgements}

The X-ray centre of Gyeongsang National University is acknowledged for providing access to the single-crystal diffractometer.

\section{References}

Anderson, J. S. (1937). Nature (London), 140, 850.

Brandenburg, K. (1998). DIAMOND. Crystal Impact GbR, Bonn, Germany.

Brese, N. E. \& O'Keeffe, M. (1991). Acta Cryst. B47, 192-197.

Brown, I. D. \& Altermatt, D. (1985). Acta Cryst. B41, 244-247.

Bruker (2009). APEX2, SADABS and SAINT. Bruker AXS Inc., Madison, Wisconsin, USA.

Day, V. W., Goloboy, J. C. \& Klemperer, W. G. (2009). Eur. J. Inorg. Chem. pp. 5079-5087.

Farrugia, L. J. (2012). J. Appl. Cryst. 45, 849-854.

Joo, H. C., Park, K. M. \& Lee, U. (1994). Acta Cryst. C50, 1659-1661.

Joo, H.-C., Park, K.-M. \& Lee, U. (2011). Acta Cryst. E67, m1801$\mathrm{m} 1802$.

Lee, U., Jang, S.-J., Joo, H.-C. \& Park, K.-M. (2003). Acta Cryst. E59, m116-m118.

Lee, U. \& Joo, H. C. (2000). Acta Cryst. C56, e311-e312.

Lee, U. \& Joo, H.-C. (2004). Acta Cryst. E60, i61-i63.

Lee, U. \& Joo, H.-C. (2010). Acta Cryst. E66, i8-i9.

Lee, U., Joo, H.-C. \& Park, K.-M. (2010). Acta Cryst. E66, i25.

Lee, U., Joo, H.-C., Park, K.-M. \& Ozeki, T. (2003). Acta Cryst. C59, $\mathrm{m} 152-\mathrm{m} 155$.

Lee, U. \& Sasaki, Y. (1994). Bull. Korean Chem. Soc., 15, 37-45.

Sheldrick, G. M. (2008). Acta Cryst. A64, 112-122.

Spek, A. L. (2009). Acta Cryst. D65, 148-155. 


\section{supporting information}

Acta Cryst. (2015). E71, 268-271 [doi:10.1107/S2056989015002601]

\section{Crystal structure of heptaguanidinium nonahydrogen bis $[\alpha-$ hexamolybdoplatinate(IV)] heptahydrate}

\section{Hea-Chung Joo, Ki-Min Park and Uk Lee}

\section{Computing details}

Data collection: APEX2 (Bruker, 2009); cell refinement: SAINT (Bruker, 2009); data reduction: SAINT; program(s) used to solve structure: SHELXS97 (Sheldrick, 2008); program(s) used to refine structure: SHELXL97 (Sheldrick, 2008); molecular graphics: ORTEP-3 for Windows (Farrugia, 2012), PLATON (Spek, 2009) and DIAMOND

(Brandenburg,1998); software used to prepare material for publication: SHELXL97.

Heptaguanidinium nonahydrogen bis[ $\alpha$-hexamolybdoplatinate(IV)] heptahydrate

\section{Crystal data}

$\left(\mathrm{CH}_{6} \mathrm{~N}_{3}\right)_{7} \mathrm{H}_{9}\left[\mathrm{PtMo}_{6} \mathrm{O}_{24}\right]_{2} \cdot 7 \mathrm{H}_{2} \mathrm{O}$

$M_{r}=2865.26$

Monoclinic, $C 2 / c$

Hall symbol: $-\mathrm{C} 2 \mathrm{yc}$

$a=31.413(10) \AA$

$b=10.073(3) \AA$

$c=23.677(7) \AA$

$\beta=119.451(14)^{\circ}$

$V=6524(3) \AA^{3}$

$Z=4$

\section{Data collection}

Bruker SMART APEXII CCD diffractometer

Radiation source: Rotating Anode Graphite multilayer monochromator Detector resolution: 10.0 pixels $\mathrm{mm}^{-1}$ $\varphi$ and $\omega$ scans

Absorption correction: multi-scan

(SADABS; Sheldrick, 2008)

$T_{\min }=0.241, T_{\max }=0.729$

Refinement

Refinement on $F^{2}$

Least-squares matrix: full

$R\left[F^{2}>2 \sigma\left(F^{2}\right)\right]=0.028$

$w R\left(F^{2}\right)=0.073$

$S=1.03$

7107 reflections

505 parameters

22 restraints
$F(000)=5416$

$D_{\mathrm{x}}=2.917 \mathrm{Mg} \mathrm{m}^{-3}$

Mo $K \alpha$ radiation, $\lambda=0.71073 \AA$

Cell parameters from 9569 reflections

$\theta=2.2-28.2^{\circ}$

$\mu=6.62 \mathrm{~mm}^{-1}$

$T=173 \mathrm{~K}$

Block, yellow

$0.30 \times 0.12 \times 0.05 \mathrm{~mm}$

56606 measured reflections

7107 independent reflections

6050 reflections with $I>2 \sigma(I)$

$R_{\text {int }}=0.033$

$\theta_{\max }=27.0^{\circ}, \theta_{\min }=1.5^{\circ}$

$h=-40 \rightarrow 36$

$k=-12 \rightarrow 12$

$l=-30 \rightarrow 30$

Primary atom site location: structure-invariant direct methods

Secondary atom site location: difference Fourier map

Hydrogen site location: difference Fourier map

$\mathrm{H}$ atoms treated by a mixture of independent and constrained refinement 
$w=1 /\left[\sigma^{2}\left(F_{\mathrm{o}}^{2}\right)+(0.0337 P)^{2}+47.9084 P\right]$

where $P=\left(F_{\mathrm{o}}^{2}+2 F_{\mathrm{c}}^{2}\right) / 3$

$(\Delta / \sigma)_{\max }=0.002$

$$
\Delta \rho_{\max }=2.50 \mathrm{e} \AA^{-3}
$$

$\Delta \rho_{\min }=-1.30 \mathrm{e} \AA^{-3}$

\section{Special details}

Geometry. All esds (except the esd in the dihedral angle between two 1.s. planes) are estimated using the full covariance matrix. The cell esds are taken into account individually in the estimation of esds in distances, angles and torsion angles; correlations between esds in cell parameters are only used when they are defined by crystal symmetry. An approximate (isotropic) treatment of cell esds is used for estimating esds involving l.s. planes.

Refinement. Refinement of $\mathrm{F}^{2}$ against ALL reflections. The weighted $\mathrm{R}$-factor $\mathrm{wR}$ and goodness of fit $\mathrm{S}$ are based on $\mathrm{F}^{2}$, conventional R-factors $R$ are based on $F$, with $F$ set to zero for negative $F^{2}$. The threshold expression of $F^{2}>2 \operatorname{sigma}\left(\mathrm{F}^{2}\right)$ is used only for calculating R-factors(gt) etc. and is not relevant to the choice of reflections for refinement. R-factors based on $\mathrm{F}^{2}$ are statistically about twice as large as those based on F, and R- factors based on ALL data will be even larger.

Fractional atomic coordinates and isotropic or equivalent isotropic displacement parameters $\left(\AA^{2}\right)$

\begin{tabular}{|c|c|c|c|c|c|}
\hline & $x$ & $y$ & $z$ & $U_{\text {iso }} * / U_{\text {eq }}$ & Occ. $(<1)$ \\
\hline Pt1 & $0.652431(6)$ & $0.176198(17)$ & $0.467608(8)$ & $0.01525(6)$ & \\
\hline Mo1 & 0.743430 & $-0.00319(4)$ & $0.57409(2)$ & $0.02093(10)$ & \\
\hline Mo2 & $0.682566(15)$ & $0.17866(4)$ & 0.624195 (19) & $0.02035(10)$ & \\
\hline Mo3 & $0.583929(15)$ & $0.34871(4)$ & $0.51759(2)$ & $0.02171(10)$ & \\
\hline Mo4 & $0.552026(14)$ & $0.34729(4)$ & $0.359683(19)$ & $0.01849(9)$ & \\
\hline Mo5 & $0.618484(14)$ & $0.17217(4)$ & $0.311229(19)$ & $0.01901(10)$ & \\
\hline Mo6 & $0.712270(14)$ & -0.01255 (4) & $0.42001(2)$ & $0.02259(10)$ & \\
\hline $\mathrm{O} 1 \mathrm{C}$ & $0.66821(11)$ & $0.0483(3)$ & $0.53959(14)$ & $0.0194(7)$ & \\
\hline $\mathrm{O} 2 \mathrm{C}$ & $0.66214(11)$ & $0.3107(3)$ & $0.53597(15)$ & $0.0187(7)$ & \\
\hline $\mathrm{H} 2$ & $0.6862(17)$ & $0.378(5)$ & $0.544(3)$ & $0.050(18)^{*}$ & \\
\hline $\mathrm{O} 3 \mathrm{C}$ & $0.58105(11)$ & $0.1962(3)$ & $0.44249(15)$ & $0.0178(7)$ & \\
\hline $\mathrm{H} 3$ & $0.568(2)$ & $0.109(3)$ & $0.439(3)$ & $0.07(2)^{*}$ & \\
\hline $\mathrm{O} 4 \mathrm{C}$ & $0.63463(11)$ & $0.3099(3)$ & $0.39634(15)$ & $0.0184(7)$ & \\
\hline $\mathrm{H} 4$ & $0.656(3)$ & $0.379(6)$ & $0.398(4)$ & $0.10(3)^{*}$ & \\
\hline $\mathrm{O} 5 \mathrm{C}$ & $0.64084(11)$ & $0.0488(3)$ & $0.39683(14)$ & $0.0191(7)$ & \\
\hline $\mathrm{O} 6 \mathrm{C}$ & $0.72274(11)$ & 0.1511 & $0.49205(15)$ & $0.0190(7)$ & \\
\hline H6 & 0.7500 & 0.2500 & 0.5000 & $0.06(3)^{*}$ & \\
\hline O7B & $0.74507(12)$ & $0.1539(3)$ & $0.62431(16)$ & $0.0229(7)$ & \\
\hline O8B & $0.61194(12)$ & $0.2010(3)$ & $0.57683(16)$ & $0.0243(7)$ & \\
\hline O9B & $0.57705(11)$ & $0.4420(3)$ & $0.44128(15)$ & $0.0226(7)$ & \\
\hline O10B & $0.55913(11)$ & $0.1971(3)$ & $0.31241(15)$ & $0.0212(7)$ & \\
\hline O11B & $0.69300(12)$ & 0.1458 & $0.35603(16)$ & $0.0235(7)$ & \\
\hline H11 & $0.713(3)$ & $0.222(7)$ & $0.362(5)$ & $0.15(4)^{*}$ & \\
\hline O12B & $0.71759(12)$ & -0.1040 & $0.49309(17)$ & $0.0262(8)$ & \\
\hline O13T & $0.80360(12)$ & 0.0093 & $0.59080(17)$ & $0.0285(8)$ & \\
\hline $\mathrm{O} 14 \mathrm{~T}$ & $0.74286(12)$ & -0.1258 & $0.62346(17)$ & $0.0305(8)$ & \\
\hline $\mathrm{O} 15 \mathrm{~T}$ & $0.68520(13)$ & 0.0508 (4) & $0.67282(16)$ & $0.0299(8)$ & \\
\hline $\mathrm{O} 16 \mathrm{~T}$ & 0.69977 (13) & 0.3193 & $0.67096(17)$ & $0.0312(8)$ & \\
\hline $\mathrm{O} 17 \mathrm{~T}$ & $0.52417(13)$ & $0.3226(4)$ & $0.49587(19)$ & $0.0371(9)$ & \\
\hline $\mathrm{O} 18 \mathrm{~T}$ & $0.60285(14)$ & $0.4814(4)$ & $0.56903(17)$ & $0.0370(9)$ & \\
\hline O19T & $0.49288(12)$ & 0.3228 & $0.34110(17)$ & $0.0275(8)$ & \\
\hline $\mathrm{O} 20 \mathrm{~T}$ & $0.55233(12)$ & 0.4783 & $0.31420(16)$ & $0.0283(8)$ & \\
\hline
\end{tabular}




\begin{tabular}{|c|c|c|c|c|c|}
\hline $\mathrm{O} 21 \mathrm{~T}$ & $0.62270(13)$ & $0.3112(4)$ & $0.27214(17)$ & $0.0292(8)$ & \\
\hline $\mathrm{O} 22 \mathrm{~T}$ & $0.60336(13)$ & $0.0433(4)$ & $0.25787(17)$ & $0.0315(8)$ & \\
\hline $\mathrm{O} 23 \mathrm{~T}$ & $0.69102(12)$ & -0.1349 & $0.36251(18)$ & $0.0304(8)$ & \\
\hline $\mathrm{O} 24 \mathrm{~T}$ & $0.77302(12)$ & 0.0093 & $0.44119(17)$ & $0.0290(8)$ & \\
\hline $\mathrm{C} 1$ & $0.5886(2)$ & $-0.1669(7)$ & $0.5871(3)$ & $0.0536(18)$ & \\
\hline N1 & $0.6167(2)$ & $-0.1725(5)$ & $0.5534(3)$ & $0.0611(18)$ & \\
\hline $\mathrm{H} 1 \mathrm{~A}$ & 0.6273 & -0.2493 & 0.5476 & $0.073^{*}$ & \\
\hline H1B & 0.6230 & -0.0991 & 0.5388 & $0.073^{*}$ & \\
\hline $\mathrm{N} 2$ & $0.57817(17)$ & $-0.2673(5)$ & $0.6092(2)$ & $0.0446(13)$ & \\
\hline $\mathrm{H} 2 \mathrm{~A}$ & 0.5879 & -0.3464 & 0.6046 & $0.053^{*}$ & \\
\hline $\mathrm{H} 2 \mathrm{~B}$ & 0.5611 & -0.2586 & 0.6293 & $0.053^{*}$ & \\
\hline N3 & 0.5678 & $-0.0394(7)$ & 0.5888 & $0.106(3)$ & \\
\hline $\mathrm{H} 3 \mathrm{~A}$ & 0.5480 & -0.0333 & 0.6052 & $0.127^{*}$ & \\
\hline $\mathrm{H} 3 \mathrm{~B}$ & 0.5748 & 0.0317 & 0.5735 & $0.127^{*}$ & \\
\hline $\mathrm{C} 2$ & 0.69992 (17) & $0.6537(5)$ & $0.7035(2)$ & $0.0252(11)$ & \\
\hline N4 & $0.7180(2)$ & $0.6254(5)$ & $0.6658(3)$ & $0.0482(14)$ & \\
\hline $\mathrm{H} 4 \mathrm{~A}$ & 0.7210 & 0.5420 & 0.6571 & $0.058 *$ & \\
\hline H4B & 0.7273 & 0.6896 & 0.6490 & $0.058 *$ & \\
\hline N5 & $0.68617(16)$ & $0.5573(4)$ & $0.7284(2)$ & $0.0310(10)$ & \\
\hline $\mathrm{H} 5 \mathrm{~A}$ & 0.6892 & 0.4741 & 0.7196 & $0.037^{*}$ & \\
\hline H5B & 0.6739 & 0.5760 & 0.7538 & $0.037^{*}$ & \\
\hline N6 & $0.69567(17)$ & $0.7785(5)$ & $0.7166(2)$ & 0.0355 (11) & \\
\hline H6A & 0.7050 & 0.8426 & 0.6999 & $0.043^{*}$ & \\
\hline H6B & 0.6835 & 0.7976 & 0.7420 & $0.043^{*}$ & \\
\hline $\mathrm{C} 3$ & $0.4036(2)$ & $0.6616(5)$ & $0.2726(3)$ & $0.0290(11)$ & \\
\hline N7 & $0.43204(15)$ & $0.5625(4)$ & $0.3073(2)$ & $0.0324(10)$ & \\
\hline H7A & 0.4546 & 0.5757 & 0.3479 & $0.039 *$ & \\
\hline H7B & 0.4284 & 0.4834 & 0.2898 & $0.039 *$ & \\
\hline N8 & $0.36976(18)$ & $0.6419(5)$ & $0.2123(2)$ & $0.0444(13)$ & \\
\hline H8A & 0.3507 & 0.7078 & 0.1894 & $0.053 *$ & \\
\hline H8B & 0.3661 & 0.5628 & 0.1947 & $0.053 *$ & \\
\hline N9 & $0.4097(3)$ & $0.7778(5)$ & 0.2998 & $0.075(2)$ & \\
\hline H9A & 0.3910 & 0.8448 & 0.2775 & $0.090^{*}$ & \\
\hline H9B & 0.4326 & 0.7894 & 0.3403 & $0.090^{*}$ & \\
\hline $\mathrm{C} 4$ & 0.5228 & $0.8412(9)$ & 0.2828 & $0.028(2)$ & 0.50 \\
\hline N10 & 0.5000 & $0.7313(6)$ & 0.2500 & $0.0368(16)$ & \\
\hline $\mathrm{H} 10 \mathrm{~A}$ & $0.4775(14)$ & $0.686(4)$ & $0.2159(13)$ & $0.044 *$ & \\
\hline N11 & 0.5000 & $0.9567(6)$ & 0.2500 & 0.0471 (19) & \\
\hline H11A & $0.5223(16)$ & $1.002(4)$ & $0.2844(14)$ & $0.057 *$ & \\
\hline N12 & $0.5653(4)$ & $0.8331(14)$ & $0.3368(6)$ & $0.039(3)$ & 0.50 \\
\hline $\mathrm{H} 12 \mathrm{~A}$ & $0.579(3)$ & $0.753(5)$ & $0.343(6)$ & $0.046^{*}$ & 0.50 \\
\hline H12B & $0.584(3)$ & $0.905(6)$ & $0.355(6)$ & $0.046^{*}$ & 0.50 \\
\hline O1W & $0.51593(15)$ & $0.6741(4)$ & $0.42228(19)$ & $0.0400(10)$ & \\
\hline H1AW & $0.5475(12)$ & $0.638(6)$ & $0.446(3)$ & $0.060 *$ & \\
\hline H1BW & $0.507(2)$ & $0.678(6)$ & $0.456(2)$ & $0.060 *$ & \\
\hline $\mathrm{O} 2 \mathrm{~W}$ & $0.52988(18)$ & $-0.0205(5)$ & $0.4254(3)$ & 0.0609 (13) & \\
\hline H2AW & $0.508(3)$ & $-0.006(8)$ & $0.3811(14)$ & $0.091 *$ & \\
\hline H2BW & $0.553(2)$ & $-0.091(6)$ & $0.435(4)$ & $0.091 *$ & \\
\hline
\end{tabular}




\begin{tabular}{|c|c|c|c|c|c|}
\hline $\mathrm{O} 3 \mathrm{~W}$ & $0.6421(2)$ & $-0.3187(6)$ & $0.4650(3)$ & $0.0764(17)$ & \\
\hline H3AW & $0.6747(13)$ & $-0.346(9)$ & $0.487(4)$ & $0.115^{*}$ & \\
\hline H3BW & $0.632(4)$ & $-0.398(6)$ & $0.441(4)$ & $0.115^{*}$ & \\
\hline $\mathrm{O} 4 \mathrm{~W}$ & $0.5802(4)$ & $0.8321(11)$ & $0.3704(5)$ & $0.036(2)$ & 0.50 \\
\hline H4AW & $0.599(4)$ & $0.911(8)$ & $0.381(6)$ & $0.055^{*}$ & 0.50 \\
\hline H4BW & $0.602(4)$ & $0.770(10)$ & $0.402(5)$ & $0.055^{*}$ & 0.50 \\
\hline
\end{tabular}

Atomic displacement parameters $\left(\AA^{2}\right)$

\begin{tabular}{|c|c|c|c|c|c|c|}
\hline & $U^{11}$ & $U^{22}$ & $U^{33}$ & $U^{12}$ & $U^{13}$ & $U^{23}$ \\
\hline Pt1 & $0.01074(9)$ & $0.01946(10)$ & $0.01597(9)$ & $-0.00051(6)$ & $0.00690(7)$ & $-0.00191(7)$ \\
\hline Mo1 & $0.01368(19)$ & $0.0186(2)$ & $0.0308(2)$ & $0.00080(15)$ & $0.01116(17)$ & $0.00340(17)$ \\
\hline Mo2 & $0.0199(2)$ & $0.0212(2)$ & $0.0193(2)$ & $-0.00066(16)$ & $0.00908(17)$ & $0.00091(16)$ \\
\hline Mo3 & $0.0190(2)$ & $0.0282(2)$ & $0.0213(2)$ & $0.00438(17)$ & $0.01255(17)$ & -0.00020 \\
\hline Mo4 & $0.01413(19)$ & $0.0230(2)$ & 0.01809 (19) & $0.00203(15)$ & $0.00773(16)$ & $0.00188(16)$ \\
\hline Mo5 & $0.0170(2)$ & $0.0242(2)$ & $0.01814(19)$ & $-0.00386(16)$ & $0.01039(17)$ & $-0.00427(16)$ \\
\hline Mo6 & $0.01442(19)$ & $0.0212(2)$ & $0.0358(2)$ & $-0.00414(16)$ & $0.01523(18)$ & $-0.01035(18)$ \\
\hline $\mathrm{O} 1 \mathrm{C}$ & $0.0188(16)$ & $0.0194(17)$ & $0.0189(15)$ & $0.0004(13)$ & $0.0083(13)$ & $-0.0001(13)$ \\
\hline $\mathrm{O} 2 \mathrm{C}$ & $0.0175(16)$ & $0.0200(17)$ & $0.0203(16)$ & $-0.0025(13)$ & 0.0107 (14) & $-0.0044(13)$ \\
\hline $\mathrm{O} 3 \mathrm{C}$ & $0.0110(15)$ & $0.0224(17)$ & $0.0212(16)$ & $0.0003(13)$ & $0.0089(13)$ & $-0.0007(13)$ \\
\hline $\mathrm{O} 4 \mathrm{C}$ & $0.0157(16)$ & $0.0218(17)$ & $0.0194(16)$ & $-0.0030(13)$ & $0.0099(13)$ & $-0.0016(13)$ \\
\hline O5C & $0.0160(15)$ & $0.0227(17)$ & $0.0195(15)$ & $-0.0018(13)$ & $0.0093(13)$ & $-0.0053(13)$ \\
\hline $\mathrm{O} 6 \mathrm{C}$ & $0.0086(14)$ & $0.0223(17)$ & $0.0225(16)$ & $0.0019(12)$ & $0.0049(13)$ & $-0.0056(13)$ \\
\hline O7B & $0.0182(16)$ & $0.0249(18)$ & $0.0228(17)$ & $-0.0009(14)$ & $0.0077(14)$ & $0.0004(14)$ \\
\hline O8B & $0.0213(17)$ & $0.0329(19)$ & $0.0235(17)$ & $-0.0006(15)$ & $0.0149(15)$ & $0.0021(15)$ \\
\hline O9B & $0.0224(17)$ & $0.0214(17)$ & $0.0241(16)$ & $0.0033(14)$ & $0.0116(14)$ & $0.0006(14)$ \\
\hline O10B & $0.0144(15)$ & $0.0283(18)$ & $0.0201(16)$ & $-0.0035(13)$ & $0.0079(13)$ & $-0.0024(14)$ \\
\hline O11B & $0.0180(16)$ & $0.0287(18)$ & $0.0271(17)$ & $-0.0031(14)$ & $0.0135(15)$ & $-0.0036(15)$ \\
\hline $\mathrm{O} 12 \mathrm{~B}$ & $0.0200(17)$ & $0.0226(18)$ & $0.041(2)$ & $-0.0029(14)$ & $0.0185(16)$ & $-0.0060(15)$ \\
\hline O13T & $0.0204(17)$ & $0.0244(18)$ & $0.038(2)$ & $-0.0017(14)$ & $0.0124(16)$ & $0.0006(15)$ \\
\hline O14T & $0.0245(18)$ & $0.0262(19)$ & $0.038(2)$ & $0.0018(15)$ & $0.0131(16)$ & $0.0075(16)$ \\
\hline O15T & $0.0318(19)$ & $0.032(2)$ & $0.0264(18)$ & $0.0031(16)$ & $0.0144(16)$ & $0.0078(16)$ \\
\hline $\mathrm{O} 16 \mathrm{~T}$ & $0.030(2)$ & $0.029(2)$ & 0.0307 (19) & $0.0001(15)$ & $0.0115(17)$ & $-0.0062(15)$ \\
\hline $\mathrm{O} 17 \mathrm{~T}$ & $0.0255(19)$ & $0.057(3)$ & $0.037(2)$ & $0.0066(17)$ & $0.0210(18)$ & $0.0066(18)$ \\
\hline $\mathrm{O} 18 \mathrm{~T}$ & $0.048(2)$ & $0.036(2)$ & $0.0266(19)$ & $0.0097(18)$ & $0.0184(18)$ & $-0.0030(17)$ \\
\hline O19T & $0.0159(16)$ & $0.033(2)$ & $0.0313(19)$ & $0.0022(14)$ & $0.0099(15)$ & $0.0011(15)$ \\
\hline $\mathrm{O} 20 \mathrm{~T}$ & $0.0261(18)$ & $0.031(2)$ & $0.0254(17)$ & $0.0023(15)$ & $0.0112(15)$ & $0.0051(15)$ \\
\hline $\mathrm{O} 21 \mathrm{~T}$ & $0.0292(19)$ & $0.038(2)$ & $0.0257(18)$ & $-0.0018(16)$ & $0.0173(16)$ & $0.0027(15)$ \\
\hline $\mathrm{O} 22 \mathrm{~T}$ & $0.0311(19)$ & $0.038(2)$ & $0.0292(18)$ & $-0.0069(17)$ & $0.0176(16)$ & $-0.0152(16)$ \\
\hline $\mathrm{O} 23 \mathrm{~T}$ & $0.0255(18)$ & $0.0244(18)$ & $0.044(2)$ & $-0.0039(15)$ & $0.0197(17)$ & $-0.0126(17)$ \\
\hline $\mathrm{O} 24 \mathrm{~T}$ & $0.0257(18)$ & $0.0280(19)$ & $0.040(2)$ & $0.0018(15)$ & $0.0208(17)$ & $-0.0045(16)$ \\
\hline $\mathrm{C} 1$ & $0.035(3)$ & $0.063(5)$ & $0.052(4)$ & $-0.005(3)$ & $0.013(3)$ & $-0.013(3)$ \\
\hline $\mathrm{N} 1$ & $0.035(3)$ & $0.035(3)$ & $0.100(5)$ & $-0.006(2)$ & $0.024(3)$ & $0.000(3)$ \\
\hline $\mathrm{N} 2$ & $0.035(3)$ & $0.049(3)$ & $0.057(3)$ & $-0.003(2)$ & $0.029(3)$ & $0.012(3)$ \\
\hline N3 & $0.134(7)$ & $0.078(5)$ & $0.185(9)$ & $-0.068(5)$ & $0.140(7)$ & $-0.066(5)$ \\
\hline $\mathrm{C} 2$ & $0.019(2)$ & $0.030(3)$ & $0.021(2)$ & $-0.002(2)$ & $0.006(2)$ & $0.000(2)$ \\
\hline $\mathrm{N} 4$ & $0.075(4)$ & $0.040(3)$ & $0.063(3)$ & $-0.011(3)$ & $0.059(3)$ & $-0.005(3)$ \\
\hline N5 & $0.038(3)$ & $0.029(2)$ & $0.035(2)$ & $-0.002(2)$ & $0.026(2)$ & $-0.003(2)$ \\
\hline
\end{tabular}




\begin{tabular}{lllllll} 
N6 & $0.038(3)$ & $0.032(3)$ & $0.038(3)$ & $0.000(2)$ & $0.021(2)$ & $0.003(2)$ \\
C3 & $0.034(3)$ & $0.030(3)$ & $0.028(3)$ & $0.004(2)$ & $0.019(2)$ & $0.005(2)$ \\
N7 & $0.031(2)$ & $0.032(2)$ & $0.026(2)$ & $0.003(2)$ & $0.0069(19)$ & $0.0025(19)$ \\
N8 & $0.038(3)$ & $0.044(3)$ & $0.036(3)$ & $0.005(2)$ & $0.006(2)$ & $0.010(2)$ \\
N9 & $0.154(7)$ & $0.029(3)$ & $0.045(3)$ & $0.016(4)$ & $0.050(4)$ & $0.004(3)$ \\
C4 & $0.022(5)$ & $0.039(6)$ & $0.031(6)$ & $-0.007(4)$ & $0.019(5)$ & $-0.002(5)$ \\
N10 & $0.033(4)$ & $0.022(3)$ & $0.045(4)$ & 0.000 & $0.012(3)$ & 0.000 \\
N11 & $0.042(4)$ & $0.019(3)$ & $0.083(6)$ & 0.000 & $0.033(4)$ & 0.000 \\
N12 & $0.035(8)$ & $0.030(6)$ & $0.043(7)$ & $0.007(5)$ & $0.013(6)$ & $0.011(7)$ \\
O1W & $0.039(2)$ & $0.055(3)$ & $0.031(2)$ & $0.0063(19)$ & $0.0207(19)$ & $0.0038(18)$ \\
O2W & $0.051(3)$ & $0.037(3)$ & $0.091(4)$ & $0.000(2)$ & $0.032(3)$ & $0.006(3)$ \\
O3W & $0.069(4)$ & $0.072(4)$ & $0.097(5)$ & $0.027(3)$ & $0.048(4)$ & $0.009(3)$ \\
O4W & $0.025(6)$ & $0.034(6)$ & $0.050(7)$ & $-0.010(4)$ & $0.018(5)$ & $0.001(6)$ \\
\hline
\end{tabular}

Geometric parameters $\left(\AA,{ }^{\circ}\right)$

\begin{tabular}{|c|c|c|c|}
\hline $\mathrm{Pt} 1-\mathrm{O} 1 \mathrm{C}$ & $1.995(3)$ & $\mathrm{O} 6 \mathrm{C}-\mathrm{O} 6 \mathrm{C}^{\mathrm{i}}$ & $2.532(6)$ \\
\hline $\mathrm{Pt} 1-\mathrm{O} 2 \mathrm{C}$ & $2.015(3)$ & $\mathrm{O} 6 \mathrm{C}-\mathrm{H} 6$ & $1.266(3)$ \\
\hline $\mathrm{Pt} 1-\mathrm{O} 3 \mathrm{C}$ & $2.027(3)$ & $\mathrm{O} 11 \mathrm{~B}-\mathrm{H} 11$ & $0.95(2)$ \\
\hline Pt1-O4C & $2.011(3)$ & $\mathrm{C} 1-\mathrm{N} 2$ & $1.254(8)$ \\
\hline $\mathrm{Pt} 1-\mathrm{O} 5 \mathrm{C}$ & $1.997(3)$ & $\mathrm{C} 1-\mathrm{N} 1$ & $1.451(10)$ \\
\hline $\mathrm{Pt} 1-\mathrm{O} 6 \mathrm{C}$ & $2.005(3)$ & $\mathrm{C} 1-\mathrm{N} 3$ & $1.451(10)$ \\
\hline Mol-O1C & $2.150(3)$ & $\mathrm{N} 1-\mathrm{H} 1 \mathrm{~A}$ & 0.8800 \\
\hline Mo1-O6C & $2.317(3)$ & $\mathrm{N} 1-\mathrm{H} 1 \mathrm{~B}$ & 0.8800 \\
\hline $\mathrm{Mo} 2-\mathrm{O} 1 \mathrm{C}$ & $2.248(3)$ & $\mathrm{N} 2-\mathrm{H} 2 \mathrm{~A}$ & 0.8800 \\
\hline $\mathrm{Mo} 2-\mathrm{O} 2 \mathrm{C}$ & $2.286(3)$ & $\mathrm{N} 2-\mathrm{H} 2 \mathrm{~B}$ & 0.8800 \\
\hline $\mathrm{Mo} 3-\mathrm{O} 2 \mathrm{C}$ & $2.307(3)$ & $\mathrm{N} 3-\mathrm{H} 3 \mathrm{~A}$ & 0.8800 \\
\hline $\mathrm{Mo} 3-\mathrm{O} 3 \mathrm{C}$ & $2.318(3)$ & N3-H3B & 0.8800 \\
\hline $\mathrm{Mo} 4-\mathrm{O} 3 \mathrm{C}$ & $2.287(3)$ & $\mathrm{C} 2-\mathrm{N} 4$ & $1.304(7)$ \\
\hline $\mathrm{Mo} 4-\mathrm{O} 4 \mathrm{C}$ & $2.327(3)$ & $\mathrm{C} 2-\mathrm{N} 5$ & $1.314(6)$ \\
\hline $\mathrm{Mo5}-\mathrm{O} 4 \mathrm{C}$ & $2.289(3)$ & $\mathrm{C} 2-\mathrm{N} 6$ & $1.317(7)$ \\
\hline Mo5-O5C & $2.178(3)$ & $\mathrm{N} 4-\mathrm{H} 4 \mathrm{~A}$ & 0.8800 \\
\hline Mo6- $\mathrm{O} 5 \mathrm{C}$ & $2.123(3)$ & $\mathrm{N} 4-\mathrm{H} 4 \mathrm{~B}$ & 0.8800 \\
\hline Mo6- $\mathrm{O} 6 \mathrm{C}$ & $2.277(3)$ & $\mathrm{N} 5-\mathrm{H} 5 \mathrm{~A}$ & 0.8800 \\
\hline Mo1-O7B & $1.965(3)$ & $\mathrm{N} 5-\mathrm{H} 5 \mathrm{~B}$ & 0.8800 \\
\hline Mo1-O12B & $1.959(3)$ & N6-H6A & 0.8800 \\
\hline $\mathrm{Mo} 2-\mathrm{O} 7 \mathrm{~B}$ & $1.978(3)$ & N6-H6B & 0.8800 \\
\hline $\mathrm{Mo} 2-\mathrm{O} 8 \mathrm{~B}$ & $1.945(3)$ & $\mathrm{C} 3-\mathrm{N} 9$ & $1.303(7)$ \\
\hline Mo3-O8B & $1.934(3)$ & $\mathrm{C} 3-\mathrm{N} 8$ & $1.310(7)$ \\
\hline Mo3-O9B & $1.952(3)$ & $\mathrm{C} 3-\mathrm{N} 7$ & $1.322(6)$ \\
\hline Mo4-O9B & $1.941(3)$ & $\mathrm{N} 7-\mathrm{H} 7 \mathrm{~A}$ & 0.8800 \\
\hline $\mathrm{Mo} 4-\mathrm{O} 10 \mathrm{~B}$ & $1.959(3)$ & $\mathrm{N} 7-\mathrm{H} 7 \mathrm{~B}$ & 0.8800 \\
\hline Mo5-O10B & $1.895(3)$ & $\mathrm{N} 8-\mathrm{H} 8 \mathrm{~A}$ & 0.8800 \\
\hline Mo5-O11B & 2.058 & $\mathrm{~N} 8-\mathrm{H} 8 \mathrm{~B}$ & 0.8800 \\
\hline Mo6-O11B & $2.075(4)$ & N9-H9A & 0.8800 \\
\hline Mo6-O12B & $1.894(4)$ & N9-H9B & 0.8800 \\
\hline Mo1-O14T & $1.706(3)$ & $\mathrm{C} 4-\mathrm{N} 12$ & $1.320(12)$ \\
\hline Mo1-O13T & $1.735(3)$ & $\mathrm{C} 4-\mathrm{N} 10$ & $1.340(10)$ \\
\hline
\end{tabular}




\begin{tabular}{|c|c|c|c|}
\hline Mo2-O15T & $1.702(3)$ & $\mathrm{C} 4-\mathrm{N} 11$ & $1.387(10)$ \\
\hline Mo2-O16T & $1.713(3)$ & $\mathrm{N} 10-\mathrm{H} 10 \mathrm{~A}$ & $0.893(17)$ \\
\hline Mo3-O17T & $1.705(4)$ & $\mathrm{N} 11-\mathrm{H} 11 \mathrm{~A}$ & $0.898(18)$ \\
\hline Mo3-O18T & $1.706(4)$ & $\mathrm{N} 12-\mathrm{H} 12 \mathrm{~A}$ & $0.90(2)$ \\
\hline Mo4-O19T & $1.704(3)$ & $\mathrm{N} 12-\mathrm{H} 12 \mathrm{~B}$ & $0.90(2)$ \\
\hline Mo4-O20T & $1.706(3)$ & $\mathrm{O} 1 \mathrm{~W}-\mathrm{H} 1 \mathrm{AW}$ & $0.94(2)$ \\
\hline Mo5-O22T & $1.708(3)$ & $\mathrm{O} 1 \mathrm{~W}-\mathrm{H} 1 \mathrm{BW}$ & $0.95(2)$ \\
\hline Mo5-O21T & $1.719(3)$ & $\mathrm{O} 2 \mathrm{~W}-\mathrm{H} 2 \mathrm{AW}$ & $0.94(2)$ \\
\hline Mo6-O23T & $1.710(3)$ & $\mathrm{O} 2 \mathrm{~W}-\mathrm{H} 2 \mathrm{BW}$ & $0.95(2)$ \\
\hline Mo6-O24T & $1.732(3)$ & $\mathrm{O} 3 \mathrm{~W}-\mathrm{H} 3 \mathrm{AW}$ & $0.93(2)$ \\
\hline $\mathrm{O} 2 \mathrm{C}-\mathrm{H} 2$ & $0.96(2)$ & $\mathrm{O} 3 \mathrm{~W}-\mathrm{H} 3 \mathrm{BW}$ & $0.94(2)$ \\
\hline $\mathrm{O} 3 \mathrm{C}-\mathrm{H} 3$ & $0.96(2)$ & $\mathrm{O} 4 \mathrm{~W}-\mathrm{H} 4 \mathrm{AW}$ & $0.95(2)$ \\
\hline $\mathrm{O} 4 \mathrm{C}-\mathrm{H} 4$ & $0.95(2)$ & $\mathrm{O} 4 \mathrm{~W}-\mathrm{H} 4 \mathrm{BW}$ & $0.95(2)$ \\
\hline $\mathrm{Mo1}-\mathrm{O} 1 \mathrm{C}-\mathrm{Mo} 2$ & $95.79(12)$ & $\mathrm{O} 21 \mathrm{~T}-\mathrm{Mo} 5-\mathrm{O} 4 \mathrm{C}$ & $86.44(14)$ \\
\hline $\mathrm{Mo} 2-\mathrm{O} 2 \mathrm{C}-\mathrm{Mo} 3$ & $93.64(11)$ & $\mathrm{O} 10 \mathrm{~B}-\mathrm{Mo5}-\mathrm{O} 4 \mathrm{C}$ & $72.56(12)$ \\
\hline $\mathrm{Mo} 4-\mathrm{O} 3 \mathrm{C}-\mathrm{Mo} 3$ & $93.75(12)$ & $\mathrm{O} 11 \mathrm{~B}-\mathrm{Mo5}-\mathrm{O} 4 \mathrm{C}$ & $85.46(12)$ \\
\hline $\mathrm{Mo5}-\mathrm{O} 4 \mathrm{C}-\mathrm{Mo} 4$ & $92.64(11)$ & $\mathrm{O} 5 \mathrm{C}-\mathrm{Mo5}-\mathrm{O} 4 \mathrm{C}$ & $72.30(12)$ \\
\hline Mo6-O5C-Mo5 & $102.87(13)$ & $\mathrm{O} 23 \mathrm{~T}-\mathrm{Mo6}-\mathrm{O} 24 \mathrm{~T}$ & $105.40(16)$ \\
\hline Mo6-O6C-Mo1 & $91.14(12)$ & $\mathrm{O} 23 \mathrm{~T}-\mathrm{Mo6}-\mathrm{O} 12 \mathrm{~B}$ & $101.61(17)$ \\
\hline Mo1-O7B-Mo2 & $111.71(15)$ & $\mathrm{O} 24 \mathrm{~T}-\mathrm{Mo6}-\mathrm{O} 12 \mathrm{~B}$ & $101.99(16)$ \\
\hline $\mathrm{Mo} 3-\mathrm{O} 8 \mathrm{~B}-\mathrm{Mo} 2$ & $119.36(16)$ & $\mathrm{O} 23 \mathrm{~T}-\mathrm{Mo6}-\mathrm{O} 11 \mathrm{~B}$ & $96.50(16)$ \\
\hline Mo4-O9B-Mo3 & $119.39(17)$ & $\mathrm{O} 24 \mathrm{~T}-\mathrm{Mo6}-\mathrm{O} 11 \mathrm{~B}$ & $89.87(15)$ \\
\hline Mo5-O10B-Mo4 & $120.02(16)$ & $\mathrm{O} 12 \mathrm{~B}-\mathrm{Mo6}-\mathrm{O} 11 \mathrm{~B}$ & $154.72(14)$ \\
\hline Mo5-O11B-Mo6 & $108.97(15)$ & $\mathrm{O} 23 \mathrm{~T}-\mathrm{Mo6}-\mathrm{O} 5 \mathrm{C}$ & $93.14(14)$ \\
\hline Mo6-O12B-Mo1 & $116.75(17)$ & $\mathrm{O} 24 \mathrm{~T}-\mathrm{Mo6}-\mathrm{O} 5 \mathrm{C}$ & $155.73(14)$ \\
\hline $\mathrm{O} 1 \mathrm{C}-\mathrm{Pt} 1-\mathrm{O} 5 \mathrm{C}$ & 99.68 (14) & $\mathrm{O} 12 \mathrm{~B}-\mathrm{Mo6}-\mathrm{O} 5 \mathrm{C}$ & $89.14(13)$ \\
\hline $\mathrm{O} 1 \mathrm{C}-\mathrm{Pt} 1-\mathrm{O} 6 \mathrm{C}$ & $84.05(13)$ & $\mathrm{O} 11 \mathrm{~B}-\mathrm{Mo6}-\mathrm{O} 5 \mathrm{C}$ & $72.28(12)$ \\
\hline $\mathrm{O} 5 \mathrm{C}-\mathrm{Pt} 1-\mathrm{O} 6 \mathrm{C}$ & $83.21(12)$ & $\mathrm{O} 23 \mathrm{~T}-\mathrm{Mo6}-\mathrm{O} 6 \mathrm{C}$ & $166.99(14)$ \\
\hline $\mathrm{O} 1 \mathrm{C}-\mathrm{Pt} 1-\mathrm{O} 4 \mathrm{C}$ & $177.35(12)$ & $\mathrm{O} 24 \mathrm{~T}-\mathrm{Mo6}-\mathrm{O} 6 \mathrm{C}$ & $87.60(14)$ \\
\hline $\mathrm{O} 5 \mathrm{C}-\mathrm{Pt} 1-\mathrm{O} 4 \mathrm{C}$ & $82.27(13)$ & $\mathrm{O} 12 \mathrm{~B}-\mathrm{Mo6}-\mathrm{O} 6 \mathrm{C}$ & $75.67(13)$ \\
\hline $\mathrm{O} 6 \mathrm{C}-\mathrm{Pt} 1-\mathrm{O} 4 \mathrm{C}$ & $97.99(13)$ & $\mathrm{O} 11 \mathrm{~B}-\mathrm{Mo6}-\mathrm{O} 6 \mathrm{C}$ & $82.71(13)$ \\
\hline $\mathrm{O} 1 \mathrm{C}-\mathrm{Pt} 1-\mathrm{O} 2 \mathrm{C}$ & $82.64(13)$ & $\mathrm{O} 5 \mathrm{C}-\mathrm{Mo6}-\mathrm{O} 6 \mathrm{C}$ & $74.21(11)$ \\
\hline $\mathrm{O} 5 \mathrm{C}-\mathrm{Pt} 1-\mathrm{O} 2 \mathrm{C}$ & $177.41(13)$ & $\mathrm{Pt} 1-\mathrm{O} 1 \mathrm{C}-\mathrm{Mo} 1$ & $104.21(13)$ \\
\hline $\mathrm{O} 6 \mathrm{C}-\mathrm{Pt} 1-\mathrm{O} 2 \mathrm{C}$ & $98.20(13)$ & $\mathrm{Pt} 1-\mathrm{O} 1 \mathrm{C}-\mathrm{Mo} 2$ & $103.95(14)$ \\
\hline $\mathrm{O} 4 \mathrm{C}-\mathrm{Pt} 1-\mathrm{O} 2 \mathrm{C}$ & $95.37(13)$ & $\mathrm{Pt} 1-\mathrm{O} 2 \mathrm{C}-\mathrm{Mo} 2$ & $101.92(13)$ \\
\hline $\mathrm{O} 1 \mathrm{C}-\mathrm{Pt} 1-\mathrm{O} 3 \mathrm{C}$ & $95.12(13)$ & $\mathrm{Pt} 1-\mathrm{O} 2 \mathrm{C}-\mathrm{Mo} 3$ & $103.51(13)$ \\
\hline $\mathrm{O} 5 \mathrm{C}-\mathrm{Pt} 1-\mathrm{O} 3 \mathrm{C}$ & $95.66(12)$ & $\mathrm{Pt} 1-\mathrm{O} 2 \mathrm{C}-\mathrm{H} 2$ & $116(4)$ \\
\hline $\mathrm{O} 6 \mathrm{C}-\mathrm{Pt} 1-\mathrm{O} 3 \mathrm{C}$ & $178.46(12)$ & $\mathrm{Mo} 2-\mathrm{O} 2 \mathrm{C}-\mathrm{H} 2$ & $113(4)$ \\
\hline $\mathrm{O} 4 \mathrm{C}-\mathrm{Pt} 1-\mathrm{O} 3 \mathrm{C}$ & $82.87(13)$ & $\mathrm{Mo} 3-\mathrm{O} 2 \mathrm{C}-\mathrm{H} 2$ & $125(4)$ \\
\hline $\mathrm{O} 2 \mathrm{C}-\mathrm{Pt} 1-\mathrm{O} 3 \mathrm{C}$ & $82.96(13)$ & $\mathrm{Pt} 1-\mathrm{O} 3 \mathrm{C}-\mathrm{Mo} 4$ & $103.59(13)$ \\
\hline O14T-Mo1-O13T & $105.87(16)$ & $\mathrm{Pt} 1-\mathrm{O} 3 \mathrm{C}-\mathrm{Mo} 3$ & $102.74(13)$ \\
\hline $\mathrm{O} 14 \mathrm{~T}-\mathrm{Mo1}-\mathrm{O} 12 \mathrm{~B}$ & $99.01(16)$ & $\mathrm{Pt} 1-\mathrm{O} 3 \mathrm{C}-\mathrm{H} 3$ & $108(4)$ \\
\hline $\mathrm{O} 13 \mathrm{~T}-\mathrm{Mo1}-\mathrm{O} 12 \mathrm{~B}$ & $98.14(15)$ & $\mathrm{Mo} 4-\mathrm{O} 3 \mathrm{C}-\mathrm{H} 3$ & $124(4)$ \\
\hline $\mathrm{O} 14 \mathrm{~T}-\mathrm{Mo1}-\mathrm{O} 7 \mathrm{~B}$ & $100.03(16)$ & $\mathrm{Mo} 3-\mathrm{O} 3 \mathrm{C}-\mathrm{H} 3$ & $122(4)$ \\
\hline $\mathrm{O} 13 \mathrm{~T}-\mathrm{Mo1}-\mathrm{O} 7 \mathrm{~B}$ & $95.86(15)$ & $\mathrm{Pt} 1-\mathrm{O} 4 \mathrm{C}-\mathrm{Mo} 5$ & $100.53(13)$ \\
\hline $\mathrm{O} 12 \mathrm{~B}-\mathrm{Mo} 1-\mathrm{O} 7 \mathrm{~B}$ & $152.25(14)$ & $\mathrm{Pt} 1-\mathrm{O} 4 \mathrm{C}-\mathrm{Mo} 4$ & $102.74(13)$ \\
\hline $\mathrm{O} 14 \mathrm{~T}-\mathrm{Mo1}-\mathrm{O} 1 \mathrm{C}$ & $93.17(14)$ & $\mathrm{Pt} 1-\mathrm{O} 4 \mathrm{C}-\mathrm{H} 4$ & $124(5)$ \\
\hline
\end{tabular}




\begin{tabular}{|c|c|}
\hline $\mathrm{O} 13 \mathrm{~T}-\mathrm{Mo} 1-\mathrm{O} 1 \mathrm{C}$ & $160.02(14)$ \\
\hline $\mathrm{O} 12 \mathrm{~B}-\mathrm{Mo} 1-\mathrm{O} 1 \mathrm{C}$ & $84.50(12)$ \\
\hline $\mathrm{O} 7 \mathrm{~B}-\mathrm{Mo1}-\mathrm{O} 1 \mathrm{C}$ & $74.48(12)$ \\
\hline $\mathrm{O} 14 \mathrm{~T}-\mathrm{Mo1}-\mathrm{O} 6 \mathrm{C}$ & $165.12(14)$ \\
\hline $\mathrm{O} 13 \mathrm{~T}-\mathrm{Mo} 1-\mathrm{O} 6 \mathrm{C}$ & $88.14(14)$ \\
\hline $\mathrm{O} 12 \mathrm{~B}-\mathrm{Mo} 1-\mathrm{O} 6 \mathrm{C}$ & $73.55(13)$ \\
\hline $\mathrm{O} 7 \mathrm{~B}-\mathrm{Mo1}-\mathrm{O} 6 \mathrm{C}$ & $83.11(12)$ \\
\hline $\mathrm{O} 1 \mathrm{C}-\mathrm{Mo1}-\mathrm{O} 6 \mathrm{C}$ & $73.54(11)$ \\
\hline $\mathrm{O} 15 \mathrm{~T}-\mathrm{Mo} 2-\mathrm{O} 16 \mathrm{~T}$ & $107.06(18)$ \\
\hline $\mathrm{O} 15 \mathrm{~T}-\mathrm{Mo} 2-\mathrm{O} 8 \mathrm{~B}$ & $98.04(15)$ \\
\hline $\mathrm{O} 16 \mathrm{~T}-\mathrm{Mo} 2-\mathrm{O} 8 \mathrm{~B}$ & $100.65(16)$ \\
\hline $\mathrm{O} 15 \mathrm{~T}-\mathrm{Mo} 2-\mathrm{O} 7 \mathrm{~B}$ & $100.70(15)$ \\
\hline $\mathrm{O} 16 \mathrm{~T}-\mathrm{Mo} 2-\mathrm{O} 7 \mathrm{~B}$ & $96.07(16)$ \\
\hline $\mathrm{O} 8 \mathrm{~B}-\mathrm{Mo} 2-\mathrm{O} 7 \mathrm{~B}$ & $149.93(14)$ \\
\hline $\mathrm{O} 15 \mathrm{~T}-\mathrm{Mo} 2-\mathrm{O} 1 \mathrm{C}$ & $94.74(15)$ \\
\hline $\mathrm{O} 16 \mathrm{~T}-\mathrm{Mo} 2-\mathrm{O} 1 \mathrm{C}$ & $156.95(15)$ \\
\hline $\mathrm{O} 8 \mathrm{~B}-\mathrm{Mo} 2-\mathrm{O} 1 \mathrm{C}$ & $83.19(13)$ \\
\hline $\mathrm{O} 7 \mathrm{~B}-\mathrm{Mo} 2-\mathrm{O} 1 \mathrm{C}$ & $72.05(12)$ \\
\hline $\mathrm{O} 15 \mathrm{~T}-\mathrm{Mo} 2-\mathrm{O} 2 \mathrm{C}$ & $163.07(14)$ \\
\hline $\mathrm{O} 16 \mathrm{~T}-\mathrm{Mo} 2-\mathrm{O} 2 \mathrm{C}$ & $88.12(15)$ \\
\hline $\mathrm{O} 8 \mathrm{~B}-\mathrm{Mo} 2-\mathrm{O} 2 \mathrm{C}$ & $71.19(12)$ \\
\hline $\mathrm{O} 7 \mathrm{~B}-\mathrm{Mo} 2-\mathrm{O} 2 \mathrm{C}$ & $84.67(12)$ \\
\hline $\mathrm{O} 1 \mathrm{C}-\mathrm{Mo} 2-\mathrm{O} 2 \mathrm{C}$ & $71.47(12)$ \\
\hline $\mathrm{O} 17 \mathrm{~T}-\mathrm{Mo} 3-\mathrm{O} 18 \mathrm{~T}$ & $106.36(19)$ \\
\hline $\mathrm{O} 17 \mathrm{~T}-\mathrm{Mo} 3-\mathrm{O} 8 \mathrm{~B}$ & $98.10(16)$ \\
\hline $\mathrm{O} 18 \mathrm{~T}-\mathrm{Mo} 3-\mathrm{O} 8 \mathrm{~B}$ & $102.14(16)$ \\
\hline $\mathrm{O} 17 \mathrm{~T}-\mathrm{Mo} 3-\mathrm{O} 9 \mathrm{~B}$ & $100.99(16)$ \\
\hline $\mathrm{O} 18 \mathrm{~T}-\mathrm{Mo} 3-\mathrm{O} 9 \mathrm{~B}$ & $97.16(16)$ \\
\hline $\mathrm{O} 8 \mathrm{~B}-\mathrm{Mo} 3-\mathrm{O} 9 \mathrm{~B}$ & $147.71(13)$ \\
\hline $\mathrm{O} 17 \mathrm{~T}-\mathrm{Mo} 3-\mathrm{O} 2 \mathrm{C}$ & $160.66(16)$ \\
\hline $\mathrm{O} 18 \mathrm{~T}-\mathrm{Mo} 3-\mathrm{O} 2 \mathrm{C}$ & $91.76(16)$ \\
\hline $\mathrm{O} 8 \mathrm{~B}-\mathrm{Mo} 3-\mathrm{O} 2 \mathrm{C}$ & $70.90(12)$ \\
\hline $\mathrm{O} 9 \mathrm{~B}-\mathrm{Mo} 3-\mathrm{O} 2 \mathrm{C}$ & $82.96(12)$ \\
\hline $\mathrm{O} 17 \mathrm{~T}-\mathrm{Mo} 3-\mathrm{O} 3 \mathrm{C}$ & $92.54(15)$ \\
\hline $\mathrm{O} 18 \mathrm{~T}-\mathrm{Mo} 3-\mathrm{O} 3 \mathrm{C}$ & $159.28(16)$ \\
\hline $\mathrm{O} 8 \mathrm{~B}-\mathrm{Mo} 3-\mathrm{O} 3 \mathrm{C}$ & $83.03(13)$ \\
\hline $\mathrm{O} 9 \mathrm{~B}-\mathrm{Mo} 3-\mathrm{O} 3 \mathrm{C}$ & $70.38(13)$ \\
\hline $\mathrm{O} 2 \mathrm{C}-\mathrm{Mo} 3-\mathrm{O} 3 \mathrm{C}$ & $70.78(11)$ \\
\hline $\mathrm{O} 19 \mathrm{~T}-\mathrm{Mo} 4-\mathrm{O} 20 \mathrm{~T}$ & $107.04(16)$ \\
\hline $\mathrm{O} 19 \mathrm{~T}-\mathrm{Mo} 4-\mathrm{O} 9 \mathrm{~B}$ & $100.65(15)$ \\
\hline $\mathrm{O} 20 \mathrm{~T}-\mathrm{Mo} 4-\mathrm{O} 9 \mathrm{~B}$ & $97.05(15)$ \\
\hline $\mathrm{O} 19 \mathrm{~T}-\mathrm{Mo} 4-\mathrm{O} 10 \mathrm{~B}$ & $99.01(15)$ \\
\hline $\mathrm{O} 20 \mathrm{~T}-\mathrm{Mo} 4-\mathrm{O} 10 \mathrm{~B}$ & $101.47(15)$ \\
\hline $\mathrm{O} 9 \mathrm{~B}-\mathrm{Mo} 4-\mathrm{O} 10 \mathrm{~B}$ & $147.60(13)$ \\
\hline $\mathrm{O} 19 \mathrm{~T}-\mathrm{Mo} 4-\mathrm{O} 3 \mathrm{C}$ & $92.83(14)$ \\
\hline $\mathrm{O} 20 \mathrm{~T}-\mathrm{Mo} 4-\mathrm{O} 3 \mathrm{C}$ & $158.72(14)$ \\
\hline $\mathrm{O} 9 \mathrm{~B}-\mathrm{Mo} 4-\mathrm{O} 3 \mathrm{C}$ & $71.24(13)$ \\
\hline $\mathrm{O} 10 \mathrm{~B}-\mathrm{Mo} 4-\mathrm{O} 3 \mathrm{C}$ & $82.34(13)$ \\
\hline
\end{tabular}

\begin{tabular}{|c|c|}
\hline $\mathrm{Mo5}-\mathrm{O} 4 \mathrm{C}-\mathrm{H} 4$ & $109(5)$ \\
\hline $\mathrm{Mo} 4-\mathrm{O} 4 \mathrm{C}-\mathrm{H} 4$ & $122(5)$ \\
\hline $\mathrm{Pt} 1-\mathrm{O} 5 \mathrm{C}-\mathrm{Mo6}$ & $103.69(13)$ \\
\hline $\mathrm{Pt} 1-\mathrm{O} 5 \mathrm{C}-\mathrm{Mo5}$ & $104.87(14)$ \\
\hline $\mathrm{Pt} 1-\mathrm{O} 6 \mathrm{C}-\mathrm{Mo6}$ & $98.17(12)$ \\
\hline $\mathrm{Pt} 1-\mathrm{O} 6 \mathrm{C}-\mathrm{Mo} 1$ & $98.14(13)$ \\
\hline $\mathrm{Pt} 1-\mathrm{O} 6 \mathrm{C}-\mathrm{O} 6 \mathrm{C}^{\mathrm{i}}$ & $120.8(2)$ \\
\hline $\mathrm{Mo6}-\mathrm{O} 6 \mathrm{C}-\mathrm{O}^{\mathrm{C}} \mathrm{C}^{\mathrm{i}}$ & $121.27(19)$ \\
\hline $\mathrm{Mol}-\mathrm{O} 6 \mathrm{C}-\mathrm{O} 6 \mathrm{C}^{\mathrm{i}}$ & $120.85(19)$ \\
\hline $\mathrm{Pt} 1-\mathrm{O} 6 \mathrm{C}-\mathrm{H} 6$ & $120.8(2)$ \\
\hline Mo6- $-\mathrm{O} 6 \mathrm{C}-\mathrm{H} 6$ & $121.27(19)$ \\
\hline Mo1-O6C-H6 & $120.85(19)$ \\
\hline $\mathrm{O} 6 \mathrm{C}-\mathrm{O} 6 \mathrm{C}-\mathrm{H} 6$ & $0.00(18)$ \\
\hline Mo5-O11B-H11 & $118(7)$ \\
\hline Mo6-O11B-H11 & $125(7)$ \\
\hline $\mathrm{N} 2-\mathrm{C} 1-\mathrm{N} 1$ & $123.5(6)$ \\
\hline $\mathrm{N} 2-\mathrm{C} 1-\mathrm{N} 3$ & $119.2(7)$ \\
\hline $\mathrm{N} 1-\mathrm{C} 1-\mathrm{N} 3$ & $117.0(6)$ \\
\hline $\mathrm{C} 1-\mathrm{N} 1-\mathrm{H} 1 \mathrm{~A}$ & 120.0 \\
\hline $\mathrm{C} 1-\mathrm{N} 1-\mathrm{H} 1 \mathrm{~B}$ & 120.0 \\
\hline $\mathrm{H} 1 \mathrm{~A}-\mathrm{N} 1-\mathrm{H} 1 \mathrm{~B}$ & 120.0 \\
\hline $\mathrm{C} 1-\mathrm{N} 2-\mathrm{H} 2 \mathrm{~A}$ & 120.0 \\
\hline $\mathrm{C} 1-\mathrm{N} 2-\mathrm{H} 2 \mathrm{~B}$ & 120.0 \\
\hline $\mathrm{H} 2 \mathrm{~A}-\mathrm{N} 2-\mathrm{H} 2 \mathrm{~B}$ & 120.0 \\
\hline $\mathrm{C} 1-\mathrm{N} 3-\mathrm{H} 3 \mathrm{~A}$ & 120.0 \\
\hline $\mathrm{C} 1-\mathrm{N} 3-\mathrm{H} 3 \mathrm{~B}$ & 120.0 \\
\hline $\mathrm{H} 3 \mathrm{~A}-\mathrm{N} 3-\mathrm{H} 3 \mathrm{~B}$ & 120.0 \\
\hline $\mathrm{N} 4-\mathrm{C} 2-\mathrm{N} 5$ & $119.7(5)$ \\
\hline $\mathrm{N} 4-\mathrm{C} 2-\mathrm{N} 6$ & $120.0(5)$ \\
\hline $\mathrm{N} 5-\mathrm{C} 2-\mathrm{N} 6$ & $120.3(5)$ \\
\hline $\mathrm{C} 2-\mathrm{N} 4-\mathrm{H} 4 \mathrm{~A}$ & 120.0 \\
\hline $\mathrm{C} 2-\mathrm{N} 4-\mathrm{H} 4 \mathrm{~B}$ & 120.0 \\
\hline $\mathrm{H} 4 \mathrm{~A}-\mathrm{N} 4-\mathrm{H} 4 \mathrm{~B}$ & 120.0 \\
\hline $\mathrm{C} 2-\mathrm{N} 5-\mathrm{H} 5 \mathrm{~A}$ & 120.0 \\
\hline $\mathrm{C} 2-\mathrm{N} 5-\mathrm{H} 5 \mathrm{~B}$ & 120.0 \\
\hline $\mathrm{H} 5 \mathrm{~A}-\mathrm{N} 5-\mathrm{H} 5 \mathrm{~B}$ & 120.0 \\
\hline $\mathrm{C} 2-\mathrm{N} 6-\mathrm{H} 6 \mathrm{~A}$ & 120.0 \\
\hline $\mathrm{C} 2-\mathrm{N} 6-\mathrm{H} 6 \mathrm{~B}$ & 120.0 \\
\hline $\mathrm{H} 6 \mathrm{~A}-\mathrm{N} 6-\mathrm{H} 6 \mathrm{~B}$ & 120.0 \\
\hline $\mathrm{N} 9-\mathrm{C} 3-\mathrm{N} 8$ & $121.1(5)$ \\
\hline $\mathrm{N} 9-\mathrm{C} 3-\mathrm{N} 7$ & $118.8(5)$ \\
\hline $\mathrm{N} 8-\mathrm{C} 3-\mathrm{N} 7$ & $120.1(5)$ \\
\hline $\mathrm{C} 3-\mathrm{N} 7-\mathrm{H} 7 \mathrm{~A}$ & 120.0 \\
\hline $\mathrm{C} 3-\mathrm{N} 7-\mathrm{H} 7 \mathrm{~B}$ & 120.0 \\
\hline $\mathrm{H} 7 \mathrm{~A}-\mathrm{N} 7-\mathrm{H} 7 \mathrm{~B}$ & 120.0 \\
\hline $\mathrm{C} 3-\mathrm{N} 8-\mathrm{H} 8 \mathrm{~A}$ & 120.0 \\
\hline $\mathrm{C} 3-\mathrm{N} 8-\mathrm{H} 8 \mathrm{~B}$ & 120.0 \\
\hline $\mathrm{H} 8 \mathrm{~A}-\mathrm{N} 8-\mathrm{H} 8 \mathrm{~B}$ & 120.0 \\
\hline
\end{tabular}




$\begin{array}{llll}\text { O19T-Mo4-O4C } & 161.33(14) & \mathrm{C} 3-\mathrm{N} 9-\mathrm{H} 9 \mathrm{~A} & 120.0 \\ \text { O20T-Mo4-O4C } & 90.53(14) & \mathrm{C} 3-\mathrm{N} 9-\mathrm{H} 9 \mathrm{~B} & 120.0 \\ \text { O9B-Mo4-O4C } & 82.92(12) & \mathrm{H} 9 \mathrm{~A}-\mathrm{N} 9-\mathrm{H} 9 \mathrm{~B} & 120.0 \\ \text { O10B-Mo4-O4C } & 70.65(12) & \mathrm{N} 12-\mathrm{C} 4-\mathrm{N} 10 & 120.6(9) \\ \text { O3C-Mo4-O4C } & 70.80(11) & \mathrm{N} 12-\mathrm{C} 4-\mathrm{N} 11 & 126.3(9) \\ \text { O22T-Mo5-O21T } & 106.73(18) & \mathrm{N} 10-\mathrm{C} 4-\mathrm{N} 11 & 112.7(7) \\ \text { O22T-Mo5-O10B } & 101.18(15) & \mathrm{C} 4-\mathrm{N} 10-\mathrm{H} 10 \mathrm{~A} & 155(2) \\ \text { O21T-Mo5-O10B } & 103.69(15) & \mathrm{C} 4-\mathrm{N} 12-\mathrm{H} 12 \mathrm{~A} & 113(7) \\ \text { O22T-Mo5-O11B } & 96.63(15) & \mathrm{C} 4-\mathrm{N} 12-\mathrm{H} 12 \mathrm{~B} & 122(8) \\ \text { O21T-Mo5-O11B } & 90.77(15) & \mathrm{H} 12 \mathrm{~A}-\mathrm{N} 12-\mathrm{H} 4 \mathrm{AW} & 106(10) \\ \text { O10B-Mo5-O11B } & 152.59(14) & \mathrm{H} 12 \mathrm{~B}-\mathrm{N} 12-\mathrm{H} 4 \mathrm{AW} & 20(10) \\ \text { O22T-Mo5-O5C } & 95.73(15) & \mathrm{H} 1 \mathrm{AW}-\mathrm{O} 1 \mathrm{~W}-\mathrm{H} 1 \mathrm{BW} & 100(6) \\ \text { O21T-Mo5-O5C } & 152.95(14) & \mathrm{H} 2 \mathrm{AW}-\mathrm{O} 2 \mathrm{~W}-\mathrm{H} 2 \mathrm{BW} & 116(8) \\ \text { O10B-Mo5-O5C } & 86.07(13) & \mathrm{H} 3 \mathrm{AW}-\mathrm{O} 3 \mathrm{H}-\mathrm{H} 3 \mathrm{BW} & 92(8) \\ \text { O11B-Mo5-O5C } & 71.47(12) & \mathrm{H} 4 \mathrm{AW}-\mathrm{O} 4 \mathrm{~W}-\mathrm{H} 4 \mathrm{BW} & 103(10) \\ \text { O22T-Mo5-O4C } & 166.58(15) & & \end{array}$

Symmetry code: (i) $-x+3 / 2,-y+1 / 2,-z+1$.

Hydrogen-bond geometry $\left(\AA,{ }^{\circ}\right)$

\begin{tabular}{|c|c|c|c|c|}
\hline$D-\mathrm{H} \cdots A$ & $D-\mathrm{H}$ & $\mathrm{H} \cdots A$ & $D \cdots A$ & $D-\mathrm{H} \cdots A$ \\
\hline $\mathrm{O} 2 C-\mathrm{H} 2 \cdots \mathrm{O} 24 T^{\mathrm{i}}$ & $0.96(2)$ & $1.61(2)$ & $2.578(5)$ & $179(6)$ \\
\hline $\mathrm{O} 3 C-\mathrm{H} 3 \cdots \mathrm{O} 2 W$ & $0.96(2)$ & $1.69(3)$ & $2.622(6)$ & $164(7)$ \\
\hline $\mathrm{O} 4 C-\mathrm{H} 4 \cdots \mathrm{O} 13 T^{\mathrm{i}}$ & $0.95(2)$ & $1.63(2)$ & $2.568(5)$ & $173(9)$ \\
\hline $\mathrm{O} 6 C-\mathrm{H} 6 \cdots \mathrm{O} 6 C^{\mathrm{i}}$ & 1.27 & 1.27 & $2.532(6)$ & 180 \\
\hline $\mathrm{O} 11 B-\mathrm{H} 11 \cdots \mathrm{O} 7 B^{\mathrm{i}}$ & $0.95(2)$ & $1.74(2)$ & $2.679(5)$ & $173(10)$ \\
\hline $\mathrm{N} 1-\mathrm{H} 1 B \cdots \mathrm{O} 1 C$ & 0.88 & 2.05 & $2.864(6)$ & 154 \\
\hline $\mathrm{N} 1-\mathrm{H} 1 A \cdots \mathrm{O} 3 W$ & 0.88 & 2.33 & $2.973(9)$ & 130 \\
\hline $\mathrm{N} 2-\mathrm{H} 2 A \cdots \mathrm{O} 18 T^{\mathrm{ii}}$ & 0.88 & 2.08 & $2.940(7)$ & 165 \\
\hline $\mathrm{N} 2-\mathrm{H} 2 B \cdots \mathrm{O} 19 T^{\mathrm{iii}}$ & 0.88 & 2.22 & $3.043(6)$ & 155 \\
\hline $\mathrm{N} 3-\mathrm{H} 3 B \cdots \mathrm{O} 8 B$ & 0.88 & 2.04 & $2.874(7)$ & 157 \\
\hline $\mathrm{N} 3-\mathrm{H} 3 A \cdots \mathrm{O} 2 W^{\text {iii }}$ & 0.88 & 2.25 & $2.979(9)$ & 140 \\
\hline $\mathrm{N} 4-\mathrm{H} 4 B \cdots \mathrm{O} 14 T^{\mathrm{iv}}$ & 0.88 & 2.09 & $2.944(6)$ & 164 \\
\hline $\mathrm{N} 4-\mathrm{H} 4 A \cdots \mathrm{O} 24 T^{\mathrm{i}}$ & 0.88 & 2.48 & $3.006(6)$ & 119 \\
\hline $\mathrm{N} 5-\mathrm{H} 5 A \cdots \mathrm{O} 16 T$ & 0.88 & 2.06 & $2.890(6)$ & 157 \\
\hline $\mathrm{N} 5-\mathrm{H} 5 B \cdots \mathrm{O} 21 T^{\mathrm{v}}$ & 0.88 & 2.18 & $2.973(5)$ & 149 \\
\hline $\mathrm{N} 6-\mathrm{H} 6 A \cdots \mathrm{O} 15 T^{\mathrm{iv}}$ & 0.88 & 2.19 & $2.894(6)$ & 136 \\
\hline $\mathrm{N} 6-\mathrm{H} 6 B \cdots \mathrm{O} 21 T^{\mathrm{v}}$ & 0.88 & 2.59 & $3.281(6)$ & 136 \\
\hline $\mathrm{N} 7-\mathrm{H} 7 B \cdots \mathrm{O} 19 T$ & 0.88 & 2.40 & $2.936(5)$ & 119 \\
\hline $\mathrm{N} 7-\mathrm{H} 7 A \cdots \mathrm{O} 1 W$ & 0.88 & 2.11 & $2.927(6)$ & 154 \\
\hline $\mathrm{N} 8-\mathrm{H} 8 B \cdots \mathrm{O} 13 T^{\mathrm{vi}}$ & 0.88 & 2.39 & $3.006(6)$ & 128 \\
\hline $\mathrm{N} 8-\mathrm{H} 8 A \cdots \mathrm{O} 23 T^{\mathrm{vii}}$ & 0.88 & 2.04 & $2.918(6)$ & 178 \\
\hline $\mathrm{N} 9-\mathrm{H} 9 A \cdots \mathrm{O} 22 T^{\mathrm{vii}}$ & 0.88 & 2.21 & $2.938(7)$ & 140 \\
\hline $\mathrm{O} 1 W-\mathrm{H} 1 A W \cdots \mathrm{O} 9 B$ & $0.94(2)$ & $2.20(5)$ & $2.916(5)$ & $132(5)$ \\
\hline $\mathrm{O} 1 W-\mathrm{H} 1 B W \cdots \mathrm{O} 17 T^{\mathrm{vii}}$ & $0.95(2)$ & $1.85(3)$ & $2.783(5)$ & $166(6)$ \\
\hline
\end{tabular}




\section{supporting information}

$\mathrm{O} 2 W-\mathrm{H} 2 B W \cdots \mathrm{O} 4 W^{\mathrm{ii}}$

$\mathrm{O} 3 W-\mathrm{H} 3 B W \cdots \mathrm{O} 9 B^{\mathrm{ii}}$

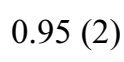

$0.94(2)$
$2.24(7)$

$2.35(8)$
$2.902(12)$

$3.029(7)$
$126(6)$

$128(8)$

Symmetry codes: (i) $-x+3 / 2,-y+1 / 2,-z+1$; (ii) $x, y-1, z$; (iii) $-x+1,-y,-z+1$; (iv) $x, y+1, z$; (v) $x,-y+1, z+1 / 2$; (vi) $x-1 / 2,-y+1 / 2, z-1 / 2$; (vii) $-x+1, y+1$, $-z+1 / 2$; (viii) $-x+1,-y+1,-z+1$. 